\title{
Orientia tsutsugamushi ankyrin repeat-containing protein family members are Type 1 secretion system substrates that traffic to the host cell endoplasmic reticulum
}

\author{
Lauren VieBrock ${ }^{1+}$, Sean M. Evans ${ }^{1+}$, Andrea R. Beyer ${ }^{1}$, Charles L. Larson ${ }^{2}$, Paul A. Beare ${ }^{2}$, Hong Ge $^{3}$, \\ Smita Singh ${ }^{1}$, Kyle G. Rodino ${ }^{1}$, Robert A. Heinzen ${ }^{2}$, Allen L. Richards ${ }^{3}$ and Jason A. Carlyon ${ }^{1 *}$ \\ ${ }^{1}$ Department of Microbiology and Immunology, Virginia Commonwealth University School of Medicine, Richmond, VA, USA \\ ${ }^{2}$ Coxiella Pathogenesis Section, Rocky Mountain Laboratories, National Institute of Allergy and Infectious Diseases, National Institutes of Health, Hamilton, MT, \\ USA \\ ${ }^{3}$ Viral and Rickettsial Diseases Department, Naval Medical Research Center, Silver Spring, MD, USA
}

Edited by:

Alfredo G. Torres, University of

Texas Medical Branch, USA

Reviewed by:

Abdu F. Azad, University of

Maryland, USA

Juan Jose Martinez, Louisiana State

University School of Veterinary

Medicine, USA

${ }^{*}$ Correspondence:

Jason A. Carlyon, Department of Microbiology and Immunology,

Virginia Commonwealth University

School of Medicine, PO Box 980678,

Richmond, VA 23298-0678, USA

e-mail: jacarlyon@vcu.edu

${ }^{\dagger}$ These authors have contributed equally to this work.
Scrub typhus is an understudied, potentially fatal infection that threatens one billion persons in the Asia-Pacific region. How the causative obligate intracellular bacterium, Orientia tsutsugamushi, facilitates its intracellular survival and pathogenesis is poorly understood. Many intracellular bacterial pathogens utilize the Type 1 (T1SS) or Type 4 secretion system (T4SS) to translocate ankyrin repeat-containing proteins (Anks) that traffic to distinct subcellular locations and modulate host cell processes. The O. tsutsugamushi genome encodes one of the largest known bacterial Ank repertoires plus T1SS and T4SS components. Whether these potential virulence factors are expressed during infection, how the Anks are potentially secreted, and to where they localize in the host cell are not known. We determined that $O$. tsutsugamushi transcriptionally expresses 20 unique ank genes as well as genes for both T1SS and T4SS during infection of mammalian host cells. Examination of the Anks' C-termini revealed that the majority of them resemble T1SS substrates. Escherichia coli expressing a functional T1SS was able to secrete chimeric hemolysin proteins bearing the C-termini of 19 of 20 O. tsutsugamushi Anks in an HlyBD-dependent manner. Thus, O. tsutsugamushi Anks C-termini are T1SS-compatible. Conversely, Coxiella burnetii could not secrete heterologously expressed Anks in a T4SS-dependent manner. Analysis of the subcellular distribution patterns of 20 ectopically expressed Anks revealed that, while 6 remained cytosolic or trafficked to the nucleus, 14 localized to, and in some cases, altered the morphology of the endoplasmic reticulum. This study identifies $O$. tsutsugamushi Anks as T1SS substrates and indicates that many display a tropism for the host cell secretory pathway.

Keywords: Rickettsia, intracellular bacteria, bacterial effector, scrub typhus, ankyrin repeat, bacterial secretion, ER-tropic effector, secretory pathway

\section{INTRODUCTION}

Scrub typhus is an acute, febrile, and potentially fatal zoonosis that is endemic to the Asia-Pacific region. One billion persons are at risk for the disease, and one million cases are estimated to occur annually. Scrub typhus can account for up to $20 \%$ of undifferentiated febrile hospitalizations in rural Asia, and unless treated with appropriate antibiotics, has an estimated $10 \%$ case fatality rate. Clinical manifestations include fever, rash, pneumonitis, disseminated vasculitis, systemic vascular collapse, and loss of cardiac function. The etiologic agent of scrub typhus is Orientia tsutsugamushi, a Gram-negative obligate intracellular bacterium of the Order Rickettsiales that is transmitted to humans during the feeding of the larval, or chigger, stage of infected trombiculid mites (Valbuena and Walker, 2012; Paris et al., 2013). The bacterium invades leukocytes at the chigger bite site. The infected leukocytes traffic to regional lymph nodes and disseminate to the peripheral vascular system. The pathogen ultimately egresses from leukocytes to infect endothelial cells of the skin and major organs (Paris et al., 2012, 2013). During the initial hours following uptake into host cells, the pathogen escapes its host cell-derived vacuole and replicates in the cytosol (Ge and Rikihisa, 2011). Considerable O. tsutsugamushi strain diversity exists (Valbuena and Walker, 2012; Paris et al., 2013), and the bacterial factors that facilitate survival in host cells are largely uncharacterized. Genomes of the Ikeda and Boryong strains, which were isolated from scrub typhus patients in Japan and Korea, respectively (Chang et al., 1990; Ohashi et al., 1996), have been sequenced and annotated (Cho et al., 2007; Nakayama et al., 2008). These genomes are valuable resources for investigations of O. tsutsugamushi molecular pathogenesis.

Ankyrin repeat-containing proteins (Anks) are key virulence factors of intracellular bacterial pathogens (Al-Khodor 
et al., 2010; Rikihisa and Lin, 2010; Voth, 2011; Jernigan and Bordenstein, 2014). These proteins contain one or more ankyrin repeats, each of which consists of a 33-residue motif that comprises the most common protein-protein interaction motif in nature. These domains form helix-turn-helix structures within proteins that mediate interactions with target proteins (AlKhodor et al., 2010). Anks are capable of binding a diversity of targets for two reasons. First, the numbers of individual ankyrin repeats within Anks vary, which in turn affects their structure and flexibility as interaction platforms. Second, the high amino acid sequence degeneracy of each individual ankyrin repeat facilitates specificity of interactions (Jernigan and Bordenstein, 2014). Anks were first thought to be exclusive to eukaryotes and shown to be involved in a variety of processes including signal transduction, vesicular trafficking, cytoskeleton integrity, and transcriptional regulation (Al-Khodor et al., 2010; Voth, 2011; Jernigan and Bordenstein, 2014). However, many intracellular bacterial species translocate Ank effectors into host cells, which traffic to distinct subcellular locales and subvert eukaryotic processes beneficial to the pathogens (Park et al., 2004; Ijdo et al., 2007; Lin et al., 2007; Pan et al., 2008; Garcia-Garcia et al., 2009; Price et al., 2009, 2010a,b, 2011; Voth et al., 2009; Lomma et al., 2010; Luhrmann et al., 2010; Mukherjee et al., 2011; Campanacci et al., 2013).

A recent assessment of 1912 bacterial genomes revealed that $51 \%$ of them encode at least one Ank, evidencing their widespread distribution among prokaryotes. The same study also concluded that the exceptionally high composition of Anks in the proteomes of obligate intracellular bacteria surpasses that of other bacterial lifestyles and is comparable to the composition of Anks of eukaryotes (Jernigan and Bordenstein, 2014). The O. tsutsugamushi Ikeda strain genome carries 47 Ank open reading frames (ORFs) (Nakayama et al., 2008), one of the highest numbers of any obligate intracellular bacterium (Jernigan and Bordenstein, 2014). Maintenance of such a large repertoire of Ank ORFs over the course of its reductive evolution as an obligate intracellular organism implies the importance of the Anks to O. tsutsugamushi pathobiology.

To exert their modulatory effects, Anks must be translocated from bacteria into host cells. Several Rickettsiales effectors, including Anks, are deployed by the Type 1 or Type 4 secretion system (T1SS or T4SS) (Lin et al., 2007; Huang et al., 2010; Wakeel et al., 2011; Kaur et al., 2012). The T1SS translocates proteins from the cytoplasm of Gram-negative bacterial pathogens to the extracellular milieu in a single step. The T1SS consists of three cell envelope proteins, each of which is required for secretion: an ATP-binding cassette (ABC) transporter localized in the inner membrane that recognizes substrates and ensures specificity of the translocation process; a membrane fusion protein (MFP) that is a periplasmic adaptor linking the cytoplasmic and outer membrane components; and an outer membrane protein (OMP) that forms a channel that remains closed until substrate recognition. Interaction of a T1SS substrate's secretion signal with the ABC triggers assembly of the apparatus and a conformational change in the OMP to yield the functional T1SS apparatus (Thomas et al., 2014).

Bacterial T1SS effectors have been shown to be toxins, proteases, lipases, heme-binding proteins, adhesins, S-layer proteins, or modulators of host cell factors involved in critical eukaryotic pathways (Zhu et al., 2009; Luo et al., 2011; Wakeel et al., 2011; Luo and Mcbride, 2012; Thomas et al., 2014). While there is no consensus T1SS substrate signal sequence, it typically comprises the C-terminal 60 residues, is rich in several amino acids (LDAVTSIF), poor in others (KHPMWC), and is not cleaved during translocation (Delepelaire, 2004; Wakeel et al., 2011; Thomas et al., 2014). Additionally, T1SS effectors tend to have acidic pIs and contain few or no cysteine residues.

The most studied T1SS is the Escherichia coli hemolysin A (HlyA) secretion system. Recognition of the HlyA C-terminal secretion signal by $\mathrm{HlyB}$ ( $\mathrm{ABC}$ transporter) triggers recruitment of HlyD (MFP) and TolC (OMP) to the membrane, followed by T1SS assembly and HlyA secretion (Thomas et al., 2014). The HlyA secretion system is a valuable tool for studying the T1SS effectors of other bacterial species, especially those that are genetically intractable. It will secrete heterologous T1SS substrates of the Rickettsiales members Ehrlichia chaffeensis (Wakeel et al., 2011) and Rickettsia typhi (Kaur et al., 2012), as well as other bacteria (Masure et al., 1990; Thompson and Sparling, 1993), when expressed in E. coli. The HlyA T1SS also translocates recombinant HlyA chimeric proteins in which the HlyA C-terminal secretion signal has been replaced by secretion signals of heterologous T1SS substrates (Zhang et al., 1993, 1995).

The T4SS is another substrate translocation channel that traverses Gram-negative bacterial cell walls (Christie et al., 2014). T4SS effectors of several important human pathogens including Coxiella burnetii, Legionella pneumophila, Helicobacter pylori, Brucella spp., and Anaplasma phagocytophilum have been shown to modulate a wide range of host cell processes (Bhatty et al., 2013). The archetypal Agrobacterium tumefaciens VirB/VirD4 T4SS consists of 11 VirB proteins and VirD4. T4SSs of many other Gram-negative bacterial species consist of homologs of most or all A. tumefaciens VirB subunits and VirD4 (Christie et al., 2014). The O. tsutsugamushi genome encodes homologs of A. tumefaciens VirB1, VirB2, VirB3, VirB4, multiple near-identical copies of VirB6, VirB8, VirB9, VirB10, and VirD4 (Cho et al., 2007; Nakayama et al., 2008). Another T4SS that is distinct from the VirB/VirD4 apparatus is the Dot/Icm system that is carried by various Gram-negative pathogens, including L. pneumophila and C. burnetii. The VirB/VirD4 and Dot/Icm T4SSs have some discernible sequence homologies to each other (Bhatty et al., 2013). They retain at least some degree of functional homology because surrogate bacterial hosts that use the Dot/Icm system can secrete VirB/VirD4 substrates (De Jong et al., 2008; Huang et al., 2010). For both VirB/VirD4 and Dot/Icm substrates, the translocation signals tend to be located in their unstructured C-termini and can include clusters of positively charged arginine residues (VirB/D4), glutamate-rich stretches called E blocks (Dot/Icm), and hydrophobic residues (Dot/Icm) (Nagai et al., 2005; Vergunst et al., 2005; Huang et al., 2011).

Given the paucity of information on O. tsutsugamushi effectors, the importance of the T1SS and T4SS to rickettsial pathogens, and the potential of Anks to serve as virulence factors, we investigated the secretion mechanism of O. tsutsugamushi Ikeda strain Anks. C. burnetii could not secrete heterologously expressed Anks in a T4SS-dependent manner, but E. coli secreted 
chimeric HlyA proteins bearing the C-termini of O. tsutsugamushi Anks in an HlyBD-dependent manner. As a first step in elucidating their pathobiological roles, we assessed the subcellular locales to where the Anks trafficked when they were ectopically expressed in mammalian cells. Strikingly, 14 out of the 20 Anks examined localized to the endoplasmic reticulum (ER). This study provides the first experimental evidence for how any O. tsutsugamushi effector can be translocated and demonstrates the potential that a large portion of the Orientia Ank armamentarium has for disrupting the host cell secretory pathway.

\section{MATERIALS AND METHODS CULTIVATION OF UNINFECTED AND O. TSUTSUGAMUSHI INFECTED HOST CELLS}

L929 mouse fibroblast cells (CCL 1 NCTC Clone 929; American Type Culture Collection [ATCC], Manassas, VA) were grown in Eagle's Minimum Essential Medium (EMEM; Quality Biological, Gaithersburg, MD) supplemented with 2.5\% (vol/vol) fetal bovine serum (FBS; Gemini Bio-Products, West Sacramento, CA) at $35^{\circ} \mathrm{C}$ in a humidified incubator with $5 \% \mathrm{CO}_{2}$. For infecting cells with O. tsutsugamushi, L929 cells were $\gamma$-irradiated with 1500 rads followed by the addition of O. tsutsugamushi at a multiplicity of infection (MOI) of 10. Following the initial infection, the infected L929 cells were maintained as described for uninfected host cells. HeLa human cervical epithelial cells (CCL-2; ATCC) were maintained in Roswell Park Memorial Institute (RPMI)1640 (Gibco, Grand Island, NY) medium supplemented with 10\% $\mathrm{FBS}$ at $37^{\circ} \mathrm{C}$ in a humidified incubator with $5 \% \mathrm{CO}_{2}$.

\section{BURNETII CULTIVATION AND INFECTION}

C. burnetii Nine Mile RSA439 (phase II, clone 4) was cultivated axenically in ACCM-2 as previously described (Omsland et al., 2011). THP-1 human monocytic leukemia cells (TIB-202; ATCC) were maintained in RPMI-1640 medium containing 10\% FBS at $37^{\circ} \mathrm{C}$ and $5 \% \mathrm{CO}_{2}$. THP-1 monocytes were differentiated into macrophage-like cells by overnight treatment with $200 \mathrm{nM}$ phorbol-12-myristate-13-acetate (PMA) followed by two washes with phosphate-buffered saline (PBS; $1.05 \mathrm{mM} \mathrm{KH}_{2} \mathrm{PO}_{4}, 155 \mathrm{mM}$ $\mathrm{NaCl}, 2.96 \mathrm{mM} \mathrm{Na}_{2} \mathrm{HPO}_{4}, \mathrm{pH} 7.2$ ) prior to infection.

\section{IN SILICO ANALYSES OF O. TSUTSUGAMUSHI ANKS}

The Protein BLAST (Basic Local Alignment Search Tool) algorithm (http://blast.ncbi.nlm.nih.gov/Blast.cgi) (Altschul et al., 1990) was used to search each O. tsutsugamushi Ank sequence against the non-redundant protein sequences database. Identification of the amino acid locations of individual ankyrin repeats within each Ank was accomplished using the Simple Modular Architecture Research Tool (SMART) algorithm (http:// smart.embl-heidelberg.de/) (Schultz et al., 1998; Ponting et al., 1999). The final C-terminal 60 amino acids of each Ank were visually screened for the presence of amino acids that are commonly found in the secretion signal sequences of T1SS effectors (LDAVTSIF) as well as those that are commonly absent (KHPMWC) (Delepelaire, 2004; Wakeel et al., 2011). The pI of each protein of interest and its C-terminus was calculated using the protein calculator algorithm (http://protcalc.sourceforge. net/).

\section{RNA ISOLATION AND RT-PCR}

Total RNA from uninfected or O. tsutsugamushi infected cells ( $\geq 90 \%$ of host cells were infected; 11 days post infection) was isolated using the RNAqueous Total RNA Isolation kit (Life Technologies, Grand Island, NY) followed by treatment with

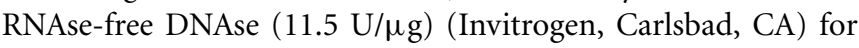
$1 \mathrm{~h}$ at $37^{\circ} \mathrm{C}$. cDNA stocks $(20 \mu \mathrm{l})$ were prepared from $1 \mu \mathrm{g}$ of DNA-free RNA using random hexamers and the iScript cDNA Synthesis kit (Biorad, Hercules, CA) followed by PCR amplification using $2 \mu \mathrm{l}$ of cDNA template and O. tsutsugamushi Ank gene-specific primers (Table A1). The thermal cycling conditions used were $94^{\circ} \mathrm{C}$ for $2 \mathrm{~min}$, followed by 35 cycles of $94^{\circ} \mathrm{C}$ for $60 \mathrm{~s}$, $55^{\circ} \mathrm{C}$ for $60 \mathrm{~s}$, and $72^{\circ} \mathrm{C}$ for $60 \mathrm{~s}$, with a final extension step of $72^{\circ} \mathrm{C}$ for $7 \mathrm{~min}$. To ensure that RNA templates were free from contaminating DNA, identical RT-PCR reactions were performed in the absence of reverse transcriptase using primers specific for the O. tsutsugamushi 16S rRNA gene (OTT_RNA006) and murine Bactin (data not shown). Representative amplicons generated using primers specific for ank5_01, ank9, ank11, ank13, and ank17 were purified using the PCRExtract Mini Kit (5 Prime, Gaithersburg, MD) and sequenced (Genewiz, South Plainfield, NJ) to verify amplicon identity.

\section{PLASMIDS FOR SUBCELLULAR LOCALIZATION STUDIES AND TISS ASSAYS}

pBMH constructs carrying O. tsutsugamushi ank genes that were codon optimized for expression in mammalian cells were synthesized by Biomatik (Wilmington, DE; Table A2) with an EcoRI restriction site and a single nucleotide that restored the reading frame upstream of the gene and a SalI restriction site downstream of the gene. pBMH vectors containing the ank genes and the recipient vectors, p3XFLAG-CMV-7.1 (Sigma-Aldrich, St. Louis, MO) and pEGFP-C1 (donated by Marci Scidmore, Cornell University, Ithaca, NY), were double-digested with 0.8 $\mathrm{U}$ each of SalI and EcoRI (New England Biolabs, Ipswich, MA) for $2 \mathrm{~h}$ at $37^{\circ} \mathrm{C}$ in EcoRI buffer (New England Biolabs), and gelpurified using the QIAquick Gel Extraction Kit (Qiagen, Valencia, CA). The restriction fragments carrying each codon-optimized ank were ligated into the digested plasmids with T4 DNA ligase (New England Biolabs) according to the manufacturer's instructions. Biomatik synthesized ank10_01 and ank14 as mammalian codon-optimized sequences and cloned them into pEGFP-C1 to generate pEGFP-Ank10 and pEGFP-Ank14, respectively.

Because p3XFLAG-CMV-7.1 and pEGFP-C1 recombinant constructs carrying the ank genes expressed each Ank as an Nterminal fusion (Table A2), we also expressed representative Anks as C-terminal 3XFLAG-tag fusions to verify that placement of the fusion tag had no bearing on the subcellular localization of ectopically expressed Anks. To this end, we PCR amplified mammalian codon-optimized ank4, ank9, and ank17 using genespecific primers that also carried a KpnI site at the $5^{\prime}$ end of the forward primer or a XbaI site at the $5^{\prime}$ end of the reverse primer (Table A3). PCR was performed using Platinum Taq HiFi DNA Polymerase (Invitrogen) and the following thermal cycling conditions: $94^{\circ} \mathrm{C}$ for $2 \mathrm{~min}$; 34 cycles of $94^{\circ} \mathrm{C}$ for $30 \mathrm{~s}, 55^{\circ} \mathrm{C}$ for $30 \mathrm{~s}$, and $68^{\circ} \mathrm{C}$ for $2 \mathrm{~min}$; followed by $68^{\circ}$ for $6 \mathrm{~min}$. The PCR products were purified using the PCR Clean-up kit ( 5 Prime) following the 
manufacturer's protocol. The p3XFLAG-CMV-14 recipient vector (Sigma-Aldrich) and the ank PCR products were each doubledigested with $0.4 \mathrm{U}$ of $\mathrm{KpnI}$ and $0.8 \mathrm{U}$ of XbaI (New England Biolabs) for $2 \mathrm{~h}$ at $37^{\circ} \mathrm{C}$, gel-purified, and ligated as described above.

To generate constructs for expressing $\mathrm{N}$-terminal His-tagged chimeric HlyA proteins in which the final $60 \mathrm{C}$-terminal amino acids of HlyA were replaced with the final 60 C-terminal amino acids of each O. tsutsugamushi Ank, we utilized the In-Fusion HD Cloning Plus kit (Clontech, Mountain View, CA) following the manufacturer's protocol to PCR amplify and clone hlyA-ank fusion PCR products into the pET19b vector (Novagen, Madison, WI). Primers for this purpose were designed using Clontech's online Primer Design Tool for In-Fusion Cloning (www.clontech.com) (Table A4). Each mammalian codon-optimized ank was PCR amplified from its respective $\mathrm{pBMH}$ construct (Table A2) using an ank gene-specific forward primer and a reverse primer that targeted the $3^{\prime}$ end of its respective ank and had an additional 17-nucleotide sequence at its $5^{\prime}$ end that was complementary to the insertion site of the pET19b vector. E. coli strain A0 34/86 was kindly provided by Peter Sebo (Institute of Microbiology, Institute of Biotechnology, Academy of Sciences of the Czech Republic, Prague, Czech Republic). The hlyA gene minus its final 180 nucleotides and stop codon was PCR amplified from DNA that had been isolated from a boiled colony of E. coli strain A0 34/86 using hlyA-specific forward and reverse primers that targeted nucleotides 4 to 2892 of $h l y A$ and had an additional 15-nucleotides at its $5^{\prime}$ end that were complementary to the first 15 nucleotides of the coding sequence for each Ank's putative Type 1 secretion signal (Table A4). PCR reactions were performed using CloneAmp HiFi PCR Premix (Clontech) and the following thermal cycling conditions: $98^{\circ} \mathrm{C}$ for $2 \mathrm{~min}$; followed by 35 cycles of $98^{\circ} \mathrm{C}$ for $10 \mathrm{~s}, 55^{\circ} \mathrm{C}$ for $15 \mathrm{~s}$, and $72^{\circ} \mathrm{C}$ for $60 \mathrm{~s}$. PCR products were resolved by gel electrophoresis, excised, purified using the QIAquick Gel Extraction Kit (Qiagen), eluted, and assayed spectrophotometrically for DNA concentration. The pET19-b vector was digested with $0.4 \mathrm{U}$ of $\mathrm{NdeI}$ at $37^{\circ} \mathrm{C}$ for $1 \mathrm{~h}$ and purified using the PCR Extract Mini Kit (5 Prime). The InFusion cloning reaction was performed according to the manufacturer's protocol and the resulting recombinant constructs were transformed into Stellar E. coli cells (Clontech). The transformants were plated on Luria-Bertani (LB) agar plates containing $100 \mu \mathrm{g} / \mathrm{ml}$ ampicillin and incubated at $37^{\circ} \mathrm{C}$ overnight. Colonies were screened via colony PCR using the $h l y A-1731 \mathrm{~F}$ forward primer (Table A4) and T7 terminator reverse primer (Integrated DNA Technologies, Coralville, IA), MyTaq Red polymerase and buffer (Bioline, Taunton, MA), and thermal cycling conditions described above. All constructs described in this section were isolated using the PerfectPrep Spin Mini Kit (5 Prime) and sequenced to verify insert integrity (Genewiz). The resulting plasmids that encode each HlyA-Ank chimeric protein consisting of HlyA lacking its final C-terminal 60 amino acids fused to the final 60 amino acids of each respective O. tsutsugamushi Ank are listed in Table A5.

\section{CyaA TRANSLOCATION ASSAY}

Mammalian codon-optimized Ank coding sequences were amplified from the plasmids listed in Table A2 using Accuprime Pfx
(Invitrogen) and the oligonucleotides listed in Table A6. The PCR products were cloned into pJB-CAT-CyaA (Voth et al., 2011) using the In-Fusion HD Cloning Plus kit (Clontech) to generate plasmids encoding each Ank N-terminally fused to CyaA (Table A7). CyaA assays were conducted as previously described (Larson et al., 2013). Briefly, PMA-differentiated THP-1 macrophages seeded in 24-well plates at a density of $5 \times 10^{5}$ cells per well were infected at a MOI of 50 with C. burnetii transformants expressing each candidate protein N-terminally fused to CyaA. After $48 \mathrm{~h}$, the cells were washed with PBS and lysed with $200 \mu \mathrm{l}$ of lysis buffer containing $50 \mathrm{mM} \mathrm{HCl}$ and $0.1 \%$ Triton X-100. Samples were boiled for $5 \mathrm{~min}$ and $400 \mu \mathrm{l}$ of $95 \%$ ethanol was added. The samples were dried under vacuum and resuspended in $400 \mu \mathrm{l}$ of assay buffer $(0.5 \mathrm{M}$ sodium acetate [pH 6.0], 0.002\% w/v bovine serum albumin). The amount of cAMP in the samples was determined with the cAMP Biotrak Enzymeimmunoassay (EIA) System (GE Healthcare, Piscataway, NJ) according to the non-acetylation procedure. Samples were measured in duplicate for each of three independent experiments. Values were reported as fold change in cAMP concentration vs. the empty vector control (CyaA only). Proteins were deemed T4SS substrates if fold change in cAMP concentration was significantly greater $(P>0.05)$ than the CyaA only control using the One-Way ANOVA statistical test, part of the Prism 6.0 software package (GraphPad, La Jolla, CA).

\section{T1SS ASSAY AND WESTERN BLOT ANALYSES}

Plasmid pLG575 (Mackman et al., 1985), which constitutively expresses E. coli HlyB and HlyD, was kindly provided by Peter Sebo (Institute of Microbiology, Institute of Biotechnology, Academy of Sciences of the Czech Republic). On day one, E. coli BL21 (DE3) cells (Bioline) were transformed with pET19-b plasmids carrying inserts that encoded His-tagged HlyA-Ank fusion proteins (Table A5) in the presence or absence of pLG575. Transformants were cultivated overnight in LB broth containing the appropriate antibiotics at $37^{\circ} \mathrm{C}$ with shaking at $250 \mathrm{rpm}$ to generate starter cultures. On day two, $1 \mathrm{ml}$ of each culture was diluted twenty-fold into fresh media and appropriate antibiotics, and the cultures were shaken at $250 \mathrm{rpm}$ at $37^{\circ} \mathrm{C}$. When the OD was between 0.6 and $1.0,0.5 \mathrm{ml}$ of each culture was retained as a non-induced control. The cultures were chilled on ice for $10 \mathrm{~min}$, after which they were induced to express proteins of interest by the addition of isopropyl $B-D$-1-thiogalactopyranoside (IPTG) to a final concentration of $1.0 \mathrm{mM}$ and shaking at $250 \mathrm{rpm}$ at $16^{\circ} \mathrm{C}$ overnight. On day three, a $0.5 \mathrm{ml}$ aliquot of each induced culture was removed, its OD determined to ensure that the E. coli had not lysed due to HlyA-Ank expression and for subsequent normalization when loading on a SDS-PAGE gel. Aliquots were pelleted at $11,000 \times \mathrm{g}$ for $10 \mathrm{~min}$, and the supernatant discarded. This saved bacterial pellet would enable us to verify that proteins of interest had been expressed. For supernatant samples, each culture was spun at $10,000 \times \mathrm{g}$ for $20 \mathrm{~min}$ at $4^{\circ} \mathrm{C}$, after which $15 \mathrm{ml}$ of the supernatant was syringe filter-sterilized through a $0.22 \mu \mathrm{m}$ filter, mixed with $15 \mathrm{ml}$ of ice-cold $40 \%$ (vol/vol) trichloroacetic acid (TCA) in acetone, and chilled at $4^{\circ} \mathrm{C}$ for $1 \mathrm{~h}$. The samples were spun at $13,000 \times \mathrm{g}$ for $15 \mathrm{~min}$, the supernatant discarded, and the pellets, which contained precipitated proteins, were each resuspended in $1 \mathrm{ml}$ of ice-cold acetone and transferred to a $1.5 \mathrm{ml}$ tube. The sample was spun at $15,000 \times \mathrm{g}$ for $5 \mathrm{~min}$ at $4^{\circ} \mathrm{C}$ 
and washed with ice-cold acetone two additional times. After pipetting to remove most of the acetone, the tube containing precipitated proteins was heated at $70^{\circ} \mathrm{C}$ for $5 \mathrm{~min}$ to evaporate residual acetone. Precipitated proteins were resuspended in $25 \mu \mathrm{l}$ of PBS.

Normalized amounts of the induced E. coli pellet samples and the entirety of the supernatant samples were resolved by SDSPAGE in 4 to $15 \%$ polyacrylamide gradient gels (Biorad) at $115 \mathrm{~V}$ for $10 \mathrm{~min}$ followed by $200 \mathrm{~V}$ for $20 \mathrm{~min}$. Proteins from the gels were transferred to nitrocellulose membrane in Towbin buffer at $100 \mathrm{~V}$ for $30 \mathrm{~min}$. The blots were blocked in 5\% (vol/vol) nonfat dry milk in tris-buffered saline plus $0.05 \%$ Tween-20 (TBS-T) for $1 \mathrm{~h}$ at room temperature. The blots were screened with mouse monoclonal antibody G3 that was specific for HlyA residues 626-673 (Rowe et al., 1994) (a kind gift from Rodney Welch, University of Wisconsin, Madison, WI) at a 1:5000 dilution in 1\% (vol/vol) non-fat dry milk in TBS-T overnight at $4^{\circ} \mathrm{C}$ with gentle rocking. The blots were washed three times in TBS-T with vigorous agitation. They were incubated with horseradish peroxidaseconjugated horse anti-mouse IgG (Cell Signaling Technology, Danvers, MA) at a 1:10,000 dilution in 1\% (vol/vol) non-fat dry milk in TBS-T for $1 \mathrm{~h}$ with rocking followed by five washes in TBS-T. Blots of E. coli pellets and supernatants were incubated with SuperSignal West Pico or SuperSignal West Dura (Thermo Scientific, Rockford, IL), respectively, and exposed to film.

\section{ECTOPIC EXPRESSION AND SUBCELLULAR LOCALIZATION STUDIES OF O. TSUTSUGAMUSHI ANKS IN MAMMALIAN HOST CELLS}

HeLa cells were seeded onto glass coverslips in 24-well plates and transfected with $0.4 \mu \mathrm{g}$ of plasmids expressing GFP- or Flagtagged Anks using Lipofectamine 2000 (Invitrogen) as directed by manufacturer. At $18-24 \mathrm{~h}$ post transfection, cells were fixed in $4 \%$ ( vol/vol) paraformaldehyde (PFA) (Electron Microscopy Science, Hatfield, PA) in PBS. Coverslips of cells expressing Flag-tagged proteins were blocked for $1 \mathrm{~h}$ at room temperature in PBS containing 5\% (vol/vol) bovine serum albumin (BSA). Next, the coverslips were stained with mouse Flag antibody (Sigma-Aldrich) at a 1:1000 dilution for $1 \mathrm{~h}$ followed by Alexa Fluor 488-conjugated goat anti-mouse IgG antibody (Invitrogen) at a dilution of 1:1000 for $1 \mathrm{~h}$. In some cases, fixed coverslips of cells ectopically expressing tagged Ank proteins were screened with chicken anti-GFP (Invitrogen), rabbit anticalnexin (Enzo Life Sciences, Farmingdale, NY), and/or rabbit anti-calreticulin (Sigma-Aldrich) at dilutions recommended by the manufacturers. Chicken anti-GFP was detected using Alexa Fluor 488-conjugated goat anti-chicken IgG (Invitrogen) at a 1:1000 dilution. Alexa Fluor 594-conjugated goat anti-rabbit IgG (Invitrogen) diluted 1:1000 was used to detect rabbit primary antibodies. Primary and secondary antibodies were diluted in PBS containing 1\% (vol/vol) BSA. Coverslips were mounted using ProLong Gold antifade plus 4',6-diamidino-2-phenylindole (DAPI) (Invitrogen) and imaged with a Zeiss LSM 700 laserscanning confocal microscope.

\section{GENERATION OF ANK4 ANTISERUM AND VALIDATION OF ITS SPECIFICITY USING A FLAG-ANK4 IMMUNOPRECIPITATION ASSAY}

Affinity purified rabbit polyclonal antiserum targeting amino acids 11-24 of Ank4_01 and of Ank4_02, which are identical paralogs, was generated by New England Peptide (Gardner, MA). HeLa cells were seeded in wells of a 6-well plate. The next day, when the cells were $90-95 \%$ confluent, $4 \mu \mathrm{g}$ of pFlagAnk4_01, pFlag-Ank9, or pFlag-BAP [bacterial alkaline phosphatase (Sigma-Aldrich)] DNA per well was transfected using Lipofectamine 2000 (Invitrogen) according to the manufacturer's directions. Between 18 and $24 \mathrm{~h}$ post transfection, $0.05 \%$ trypsin-EDTA (Invitrogen) was added to each well to promote cellular detachment and the cells were lysed in $300 \mu \mathrm{l}$ of lysis buffer $[20 \mu \mathrm{M}$ Tris $\mathrm{pH} 7.4,0.5 \mathrm{M} \mathrm{NaCl}, 0.7 \%$ Tween-20, with EDTA-free protease inhibitor (Roche Diagnostics $\mathrm{GmBH}$, Mannheim, Germany)] for $40 \mathrm{~min}$ on ice. Insoluble proteins were pelleted at $10,000 \times \mathrm{g}$ for $10 \mathrm{~min}$ at $4^{\circ} \mathrm{C}$ and discarded. Anti-Flag affinity gel beads (Sigma-Aldrich) were incubated with the cell lysates overnight at $4^{\circ} \mathrm{C}$ with rotation to precipitate Flag-tagged proteins. Resin was washed 4 times in lysis buffer prior to elution of proteins with $30 \mathrm{ul}$ of $2 \mathrm{X}$ SDS sample buffer heated to $100^{\circ} \mathrm{C}$. Samples were resolved by SDS-PAGE and transferred to nitrocellulose. The Western blots were screened with Ank4 antiserum (1:1000) or Flag antibody (1:1000; Sigma-Aldrich) followed by horseradish peroxidase (HRP)-conjugated goat anti-rabbit IgG (1:10,000, Cell Signaling Technology).

\section{IMMUNOFLUORESCENCE DETECTION OF ANK4 EXPRESSED BY 0. TSUTSUGAMUSHI IN INFECTED HOST CELLS}

L929 cells were seeded onto glass coverslips in 24-well plates and treated with $0.4 \mu \mathrm{g} / \mathrm{ml}$ daunomycin (Sigma-Aldrich) followed by the addition of media laden with O. tsutsugamushi organisms that had been naturally released from infected L929 cells. Cells were fixed in $4 \%$ (vol/vol) PFA in PBS. Cells were methanol permeabilized for $30 \mathrm{~s}$ and blocked for $1 \mathrm{~h}$ at room temperature in PBS containing 5\% ( vol/vol) BSA. Next, the coverslips were incubated with fluorescein isothiocyanate-conjugated rat anti-O. tsutsugamushi serum (undiluted) and Ank4 antiserum $(1: 100)$ for $1 \mathrm{~h}$ at room temperature followed by incubation with Alexa Fluor 594-conjugated goat anti-rabbit IgG (1:1000; Invitrogen). Primary and secondary antibodies were diluted in PBS containing 5\% and $1 \%$ (vol/vol) BSA, respectively. Coverslips were mounted using ProLong Gold antifade plus DAPI and imaged with a Zeiss LSM 700 laser-scanning confocal microscope.

\section{RESULTS}

\section{THE O. TSUTSUGAMUSHI GENOME ENCODES ANKS THAT DISPLAY CHARACTERISTICS OF T1SS SUBSTRATES}

The O. tsutsugamushi Ikeda strain genome (NCBI accession number NC_010793.1) encodes 38 ank ORFs and 9 ank pseudogenes (Nakayama et al., 2008) that are distributed throughout the chromosome. SMART (Simple Modular Architecture Research Tool) analysis revealed that each carries up to 9 ankyrin repeats (Table 1). Eighteen of the 38 ank ORFs exist as multiple identical or near-identical paralogs and 12 occur as single copy genes. Ank18 carries no ankyrin repeats but is homologous to the non-ankyrin repeat portion of Boryong Ank1u7 (OTBS_1195), which does contain an ankyrin repeat domain (Cho et al., 2007), and was therefore included in this study. We selected a subset of the 38 full-length Anks that were substantially diverse in 
Table 1 | Orientia tsutsugamushi lkeda strain Anks.

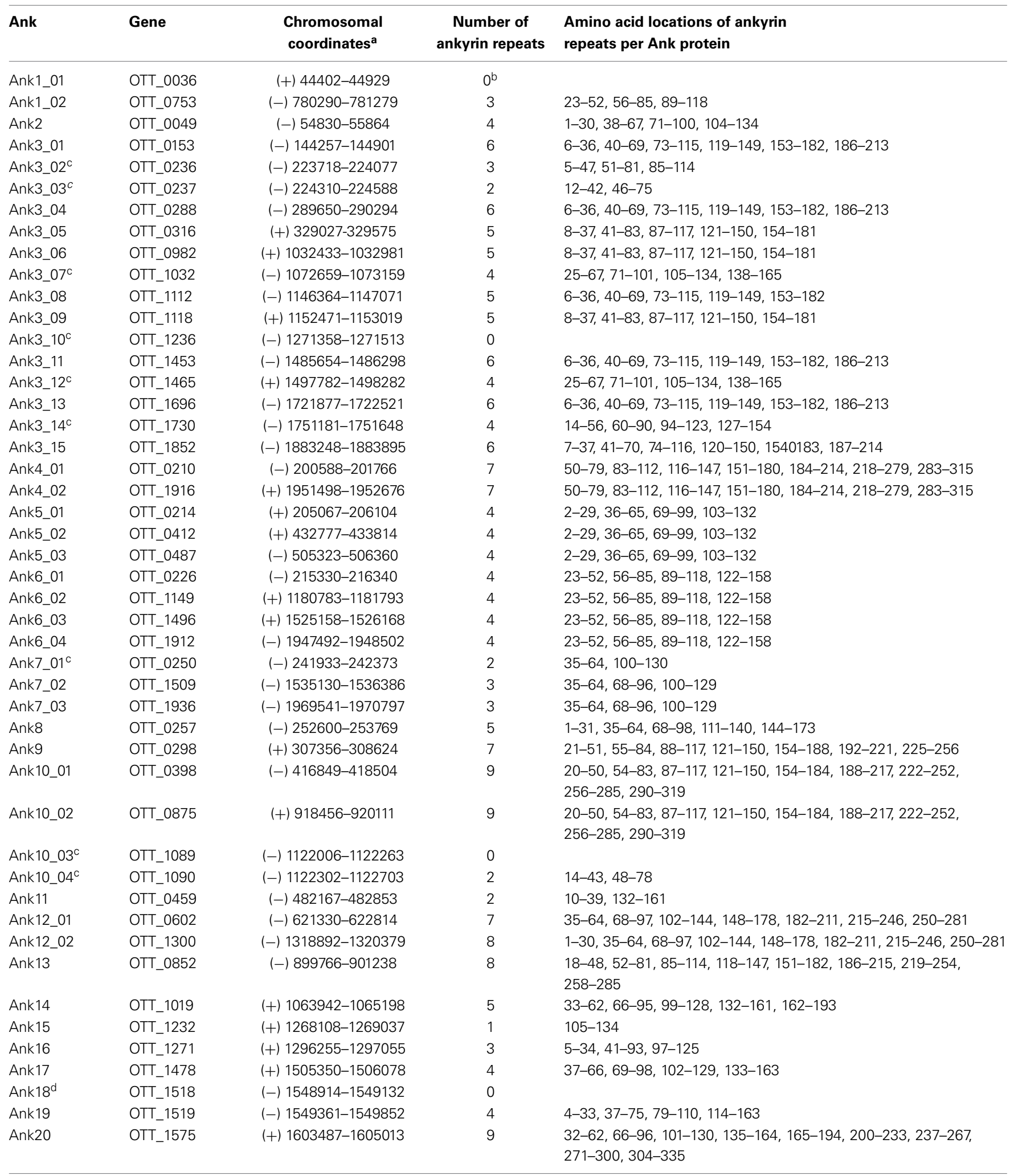

${ }^{a}(+)$ and (-) refer to the sense and antisense DNA strands, respectively.

${ }^{b}$ Ank1_01 is homologous to the non-ankyrin repeat-containing portions of Ank1_02.

c These ORFs are annotated as probable pseudogenes by Nakayama et al. (2008).

d Ank18 carries no ankyrin repeats, but exhibits homology to the non-ankyrin repeat portion of its homolog in the O. tsutsugamushi Boryong strain, Ank1u7 (OTBS_1195; Cho et al., 2007). 
sequence such that they could be differentiated from each other by PCR. We chose all of the single copy Anks and one representative member of each multi-copy Ank group to arrive at a total of 20 Anks (Table 2). The selected Anks displayed characteristics of T1SS effectors, especially those encoded by other rickettsial pathogens (Table 3) (Delepelaire, 2004; Wakeel et al., 2011; Kaur et al., 2012; Thomas et al., 2014), but not T4SS substrates. The percentage of LDAVTSIF residues occurring within the 60 C-terminal amino acids of each Ank ranged from 37 to $63 \%$. The pIs of 13 of the 20 full-length Anks were acidic, ranging between 4.6 and 6.3. Ank7_02, Ank10_01, and Ank15 had overall pIs of 7.3, 6.85, and 7.2, but had C-termini with pIs of 4.3, 5.0, and 4.2, respectively. All of the Anks had relatively few cysteines. Thus, O. tsutsugamushi Anks may be T1SS substrates.

\section{TSUTSUGAMUSHI TRANSCRIPTIONALLY EXPRESSES ANK, T1SS, AND T4SS GENES DURING INFECTION OF MAMMALIAN HOST CELLS}

The Ikeda and Boryong O. tsutsugamushi genomes encode VirB and VirD4 T4SS components (Cho et al., 2007; Nakayama et al., 2008). They also encode the T1SS components HlyB and HlyD which are sometimes annotated as AprD (alkaline protease secretion ATP-binding protein) and AprE (alkaline protease secretion protein), respectively, in other bacterial species (Lin et al., 2009; Kaur et al., 2012; Thomas et al., 2014) and are annotated as such in O. tsutsugamushi (Nakayama et al., 2008). In silico analyses of the O. tsutsugamushi T1SS components OTT_0076 (TolC),

Table 2 | O. tsutsugamushi Anks selected for codon optimization and further analyses.

\begin{tabular}{ll}
\hline Ank & \% Identity with its paralog(s) \\
\hline Ank1_02 & Ank1_01 (43.2\%) \\
Ank2 & Single copy \\
Ank3_08 & Ank3_01 (83.4\%), Ank3_04 (84.7\%), Ank3_05 (69.8\%), \\
& Ank3_06 (69.8\%), Ank3_09 (69.8\%), Ank3_11 (81.3\%), \\
& Ank3_13 (84.7\%), Ank3_15 (80.9\%) \\
Ank4_01 & Ank4_02 (100\%) \\
Ank5_01 & Ank5_02 (100\%), Ank5_03 (100\%) \\
Ank6_02 & Ank6_01 (99.1\%), Ank6_03 (100\%), Ank6_04 (100\%) \\
Ank7_02 & Ank7_03 (100\%) \\
Ank8 & Single copy \\
Ank9 & Single copy \\
Ank10_01 & Ank10_02 (100\%) \\
Ank11 & Single copy \\
Ank12_01 & 12_02 (76.4\%) \\
Ank13 & Single copy \\
Ank14 & Single copy \\
Ank15 & Single copy \\
Ank16 & Single copy \\
Ank17 & Single copy \\
Ank18 & Single copy \\
Ank19 & Single copy \\
Ank20 & Single copy \\
\hline
\end{tabular}

a Pseudogenes were excluded.
OTT_1107 (AprE), and OTT_1108 (AprD) revealed that they had 22,27 , and $41 \%$ amino acid identity to E. coli TolC, HlyD, and HlyB, respectively.

To determine if O. tsutsugamushi transcribes T1SS and T4SS component genes and any of the 20 representative ank genes during infection, total RNA isolated from infected L929 cells was subjected to reverse transcriptase (RT)-PCR using genespecific primers. Reactions performed in the absence of RT with primers specific for the O. tsutsugamushi $16 S$ rRNA gene and host $\beta$-actin yielded no PCR product (data not shown). Thus, the RNA templates were DNA-free. Reactions containing O. tsutsugamushi DNA or that lacked cDNA template were positive and negative controls, respectively. O. tsutsugamushi $16 \mathrm{~S}$ rRNA primers were used to validate the amplification conditions. All ank, T1SS component, and T4SS component transcripts except for one of the five copies of virB6 (OTT_1404) were detected in the reactions that contained RT (Figure 1). Sequencing was used to confirm the identities of representative amplicons (data not shown). Thus, O. tsutsugamushi expresses genes encoding Ank, T1SS, and T4SS components during infection of mammalian host cells.

\section{ASSAY FOR EVALUATING HIYBD-DEPENDENT SECRETION OF POTENTIAL T1SS SUBSTRATES}

We next sought to determine how O. tsutsugamushi Anks are potentially translocated. However, due to the pathogen's obligatory intracellular nature, no knock out-complementation system exists to directly assess the mechanism by which they are secreted. The Anks share features with T1SS substrates, including the presence of T1SS-like secretion signals in their C-termini. Accordingly, we evaluated O. tsutsugamushi Anks as putative T1SS effectors using the approach that previously verified $E$. coli strains K-12 or C600 could secrete recombinant forms of full-length E. chaffeensis or R. typhi T1SS candidate substrates in a TolCdependent manner, respectively (Wakeel et al., 2011; Kaur et al., 2012). However, when full-length O. tsutsugamushi Anks were tested, they were toxic to E. coli $\mathrm{C} 600$, as the bacteria grew poorly or lysed regardless of inducer concentration, length of induction time, or induction temperature (data not shown). Also, for those E. coli C600 transformants that did not lyse, the Ank proteins were detected in the supernatants of both C600 and the C600 TolC-deficient mutant, which implied leaky secretion (data not shown). Thus, the TolC-dependent method using E. coli C600 was unsuitable for assessing O. tsutsugamushi Anks as potential T1SS substrates.

As an alternative, we developed an assay in which the C-terminal 60 amino acids of HlyA containing the T1SS secretion signal were replaced with the $60 \mathrm{C}$-terminal residues of $O$. tsutsugamushi Anks and tested for secretion in an HlyBD-dependent manner. This approach is outlined in Figure $\mathbf{2}$ and was based on a report that the C-terminus of Mannheimia haemolytica (formerly Pasteurella hemolytica) leukotoxin (LktA), a T1SS effector (Davies et al., 2002), would functionally replace the T1SS secretion signal of E. coli HlyA (Zhang et al., 1993, 1995). Moreover, our approach avoided the leaky TolC-independent secretion observed for O. tsutsugamushi Anks expressed in the C600/C600 TolCdeficient mutant system, as T1SS substrates are not directed to 


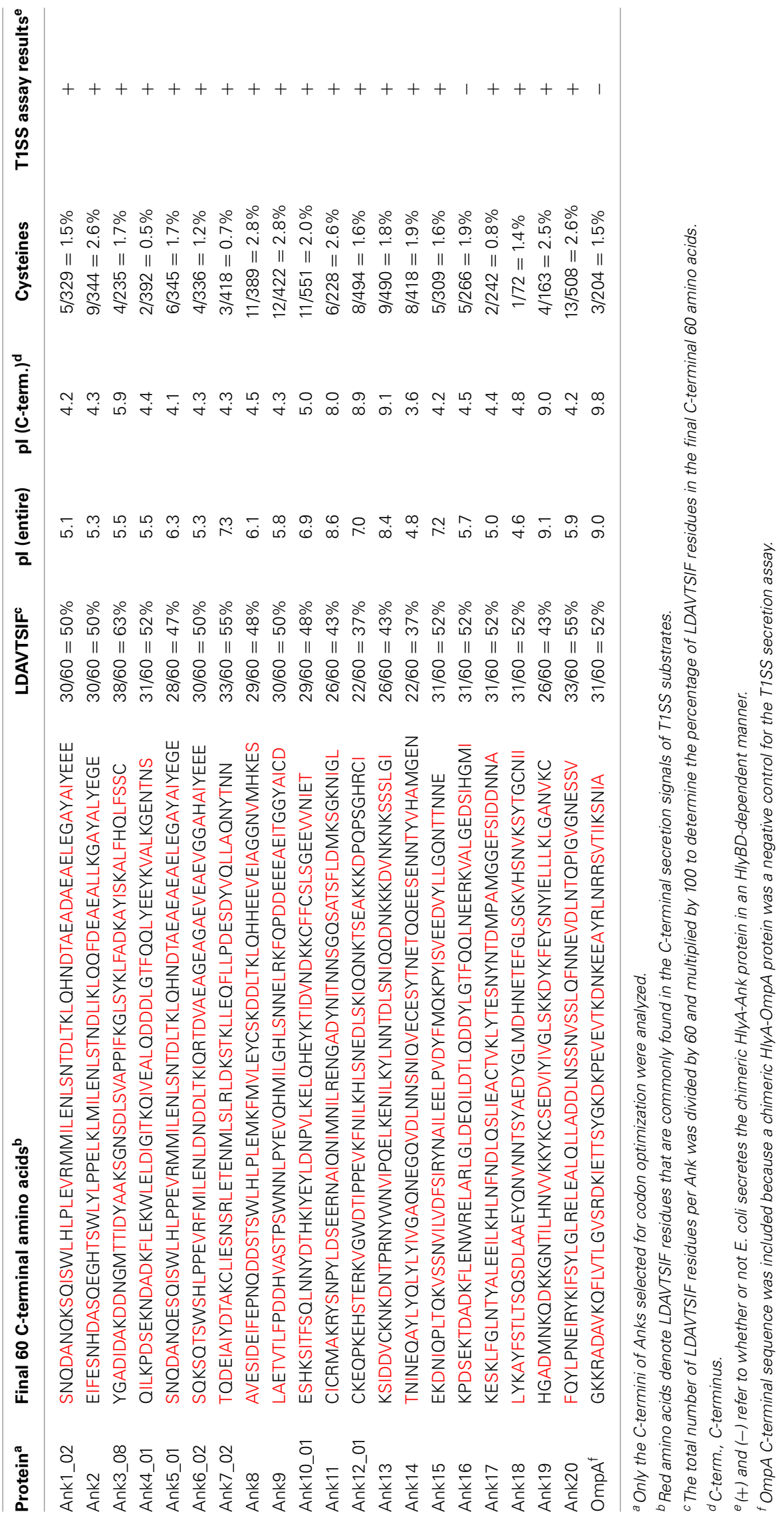




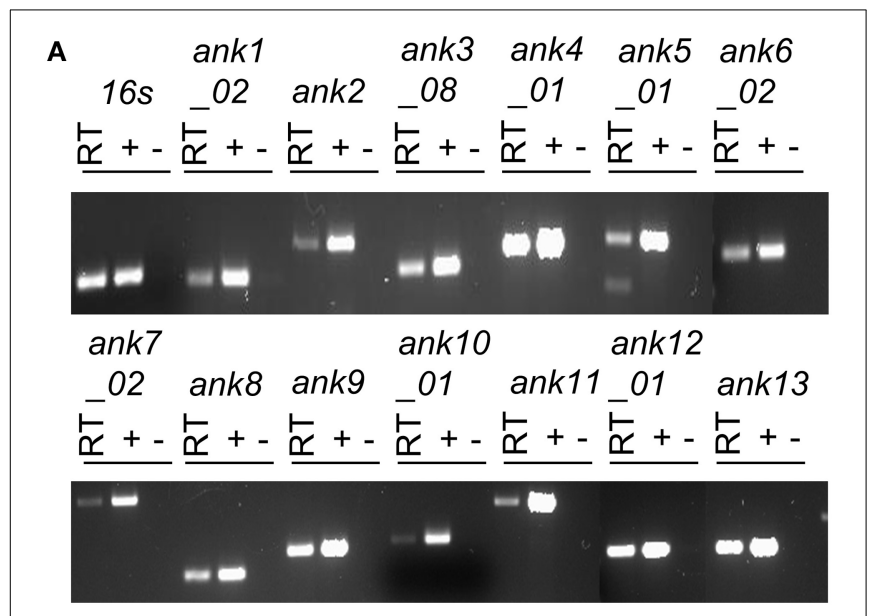

ank14 ank15 ank16 ank17 ank18 ank19 ank20

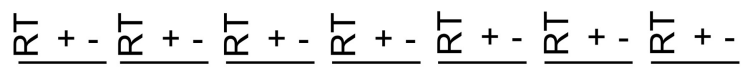

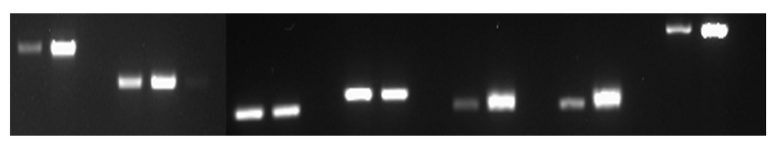

B

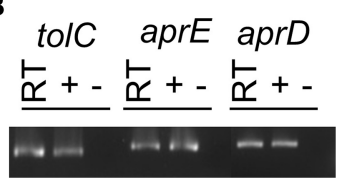

C

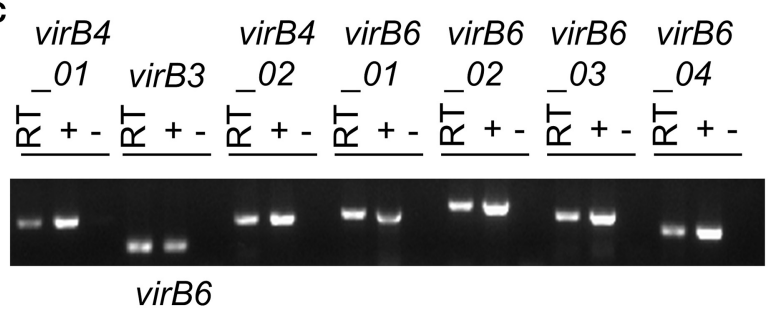

virB8 _05 virD4 virB11 virB10 virB9 $\underline{\leftarrow}+-\stackrel{\leftarrow}{\underline{\alpha}}+-\stackrel{\leftarrow}{\underline{\alpha}}+-\stackrel{\leftarrow}{\underline{\alpha}}+-\stackrel{\leftarrow}{\underline{\alpha}}+-\stackrel{\leftarrow}{\underline{\alpha}}+$

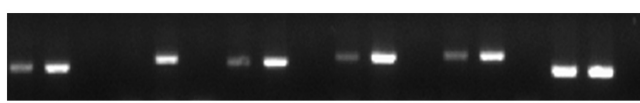

FIGURE $1 \mid$ O. tsutsugamushi transcriptionally expresses Ank, T1SS, and T4SS genes during infection of mammalian host cells. RT-PCR using primers targeting genes encoding $O$. tsutsugamushi Anks (A), T1SS components (B), T4SS components (C), and the 16S rRNA gene (infection control) (A) was performed on DNA-free total RNA isolated from infected L929 cells (RT lanes). O. tsutsugamushi genomic DNA and water served as positive $(+)$ and negative (-) controls, respectively. Results are representative of two experiments with similar results.

the TolC outer membrane channel in the absence of HlyB and HlyD (Thomas et al., 2014). E. coli strain BL21 (DE3) carries a chromosomal copy of tolC, but lacks $h l y B$ and $h l y D$. This strain was complemented with plasmid pLG575 (Mackman et al., 1985) to constitutively express HlyB and HlyD and thereby reconstitute the T1SS.
To validate the system, we tested the ability of E. coli BL21 (DE3) to secrete HlyA and a chimeric HlyA protein with its C-terminal 60 amino acids replaced with the LktA C-terminal secretion signal (HlyA-LktA) (Zhang et al., 1993) in an HlyBDdependent manner. E. coli expressing HlyA lacking the C-terminal secretion signal (HlyA $\Delta$ 965-1024) served as a negative control. Following IPTG induction of HlyA protein expression, the supernatants were Western-blotted and screened with HlyA antibody for the presence of secreted HlyA proteins (Rowe et al., 1994). HlyA is a $107-\mathrm{kDa}$ protein that after being produced quickly breaks down into a series of fragments that migrate with apparent molecular weights of $\sim 70-100 \mathrm{kDa}$ (Aldick et al., 2009). Bands corresponding to the expected sizes for full-length HlyA and its breakdown products, HlyA $\Delta 965-1024$, and HlyA-LktA were detected in the E. coli cell pellets (Figures 3A,B), thereby confirming their expression in an IPTG-inducible manner, as they were absent from non-induced samples (data not shown). Consistent with it lacking a T1SS secretion signal, HlyA $\Delta 965-1024$ was not detected in the supernatant from E. coli that also expressed HlyB and HlyD (Figure 3A). Full-length HlyA and chimeric HlyA-LktA bands were only detected in supernatants of $E$. coli that expressed HlyB and HlyD (Figures 3A,B), validating that E. coli BL21 (DE3) having a functionally reconstituted T1SS can be used as a tool to screen T1SS candidates.

\section{E. COLI SECRETES CHIMERIC HIYA PROTEINS BEARING THE C-TERMINI OF 0. TSUTSUGAMUSHI ANKS IN AN HIYBD-DEPENDENT MANNER}

To evaluate the 20 Anks of interest as potential T1SS substrates, we assessed if swapping the final 60 amino acids of HlyA with the $60 \mathrm{C}$-terminal residues of each Ank would permit secretion of HlyA-Ank chimeras in an HlyBD-dependent manner. For these assays, HlyA and HlyA $\Delta 965-1024$ served as positive and negative controls, respectively. As an additional negative control to ensure that any secretion observed for HlyA-Ank proteins was specific to the inclusion of a T1SS-compatible sequence, we assayed a chimeric protein in which the HlyA C-terminal sequence was replaced with the $60 \mathrm{C}$-terminal amino acids of O. tsutsugamushi outer membrane protein A (HlyA-OmpA). The OmpA C-terminus is similar in LDAVTSIF composition $(51.7 \%)$ to the C-termini of O. tsutsugamushi Anks and rickettsial effectors (Table 3) (Wakeel et al., 2011; Kaur et al., 2012), but is a cell envelope protein and, as such, should not be secreted. Following induction, all HlyA-Ank proteins and HlyA-OmpA were expressed, as HlyA antibody detected bands of the expected sizes for HlyA and/or its cleavage products in the E. coli cell pellets (Figure 4). With the exception of HlyA-Ank16 and HlyA-OmpA, all chimeric HlyA proteins were secreted in an HlyBD-dependent manner. The signals for secreted HlyA-Ank7_02, -Ank8, -Ank11, -Ank12_01, -Ank14, and -Ank19 were not as robust as for the other secreted HlyA-Anks. These data demonstrate that nearly all of the O. tsutsugamushi Anks bear C-terminal sequences that mediate heterologous recognition and secretion by the E. coli T1SS.

\section{THE C. BURNETII DOT/ICM TUSS CANNOT HETEROLOGOUSLY SECRETE 0. TSUTSUGAMUSHI ANKS}

Based on the HlyA-Ank secretion assay results and because of their C-terminal sequence characteristics, we rationalized that the 


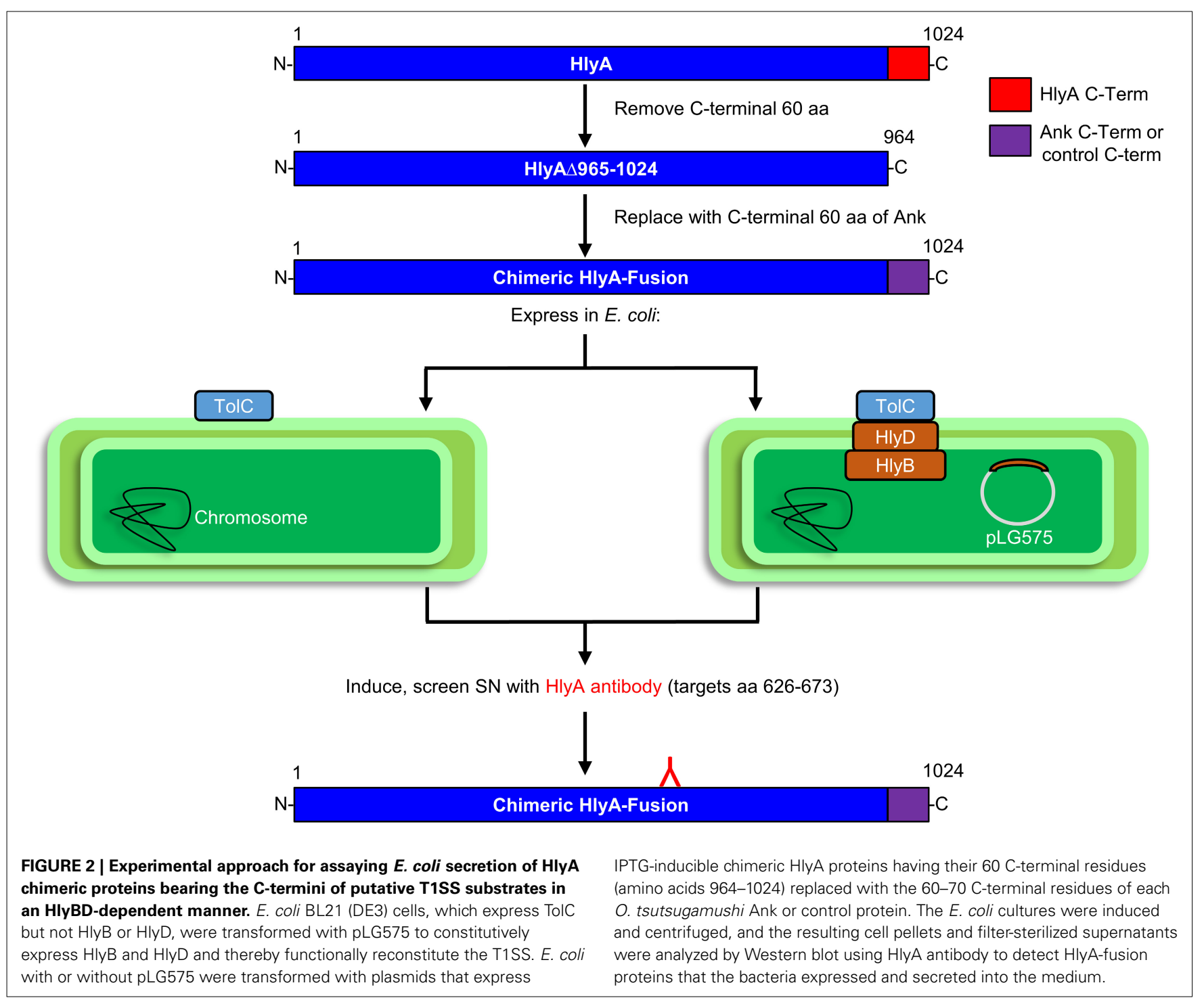

O. tsutsugamushi Anks are not T4SS effectors. To validate this hypothesis, each of the 20 Anks of interest was N-terminally fused to the enzymatic reporter, Bordetella pertussis adenylate cyclase (CyaA) and expressed in the surrogate host, C. burnetii, which uses its Dot/Icm secretory apparatus to translocate T4SS substrates (Beare et al., 2011). Surrogate hosts that use the Dot/Icm system will secrete heterologously expressed VirB/VirD4 effectors, including the Rickettsiales effector, A. phagocytophilum AnkA (De Jong et al., 2008; Huang et al., 2010). THP-1 cells that had been differentiated into macrophage-like cells were infected with C. burnetii transformants expressing CyA-fusions of O. tsutsugamushi Anks, CyaA-CvpA (Coxiella vacuolar protein A; bona fide Dot/Icm effector; positive control) (Larson et al., 2013), or CyaA alone (negative control) and changes in host cAMP levels were quantified. Expression of each Cya-fusion protein in infected host cells was confirmed by Western blot analysis (data not shown). cAMP levels were considerably elevated relative to that in C. burnetii expressing CyaA alone when host cells were infected with $C$. burnetii expressing CyaA-CvpA, but not any
CyaA-Ank (Figure 5). As expected, cAMP levels were low for host cells infected with transformants of a C. burnetii T4SS-defective DotA mutant. Thus, heterologously expressed O. tsutsugamushi Anks cannot be translocated by the C. burnetii Dot/ Icm T4SS.

\section{ECTOPICALLY EXPRESSED O. TSUTSUGAMUSHI ANKS EXHIBIT DIVERSE SUBCELLULAR LOCALIZATION PATTERNS}

Upon secretion into host cells, bacterial effectors traffic to distinct subcellular locales where they exert their modulatory functions. As a first step in gaging the subcellular locations to where O. tsutsugamushi Anks potentially traffic, confocal microscopy was used to examine green fluorescent protein (GFP)-tagged Anks in HeLa cells. GFP-tagged Ank1_02, Ank2, Ank3_08, Ank4_01, Ank5_01, Ank6_02, Ank8, Ank10_01, Ank14, and Ank19 exhibited aggregative, perinuclear fluorescence patterns (Figure 6A). GFP-tagged Ank7_02, Ank9, Ank12_01, Ank15, Ank16, and Ank20 displayed vesicular and/or cytosolic aggregative staining patterns. GFP-Ank11 exhibited a reticulate distribution, while 


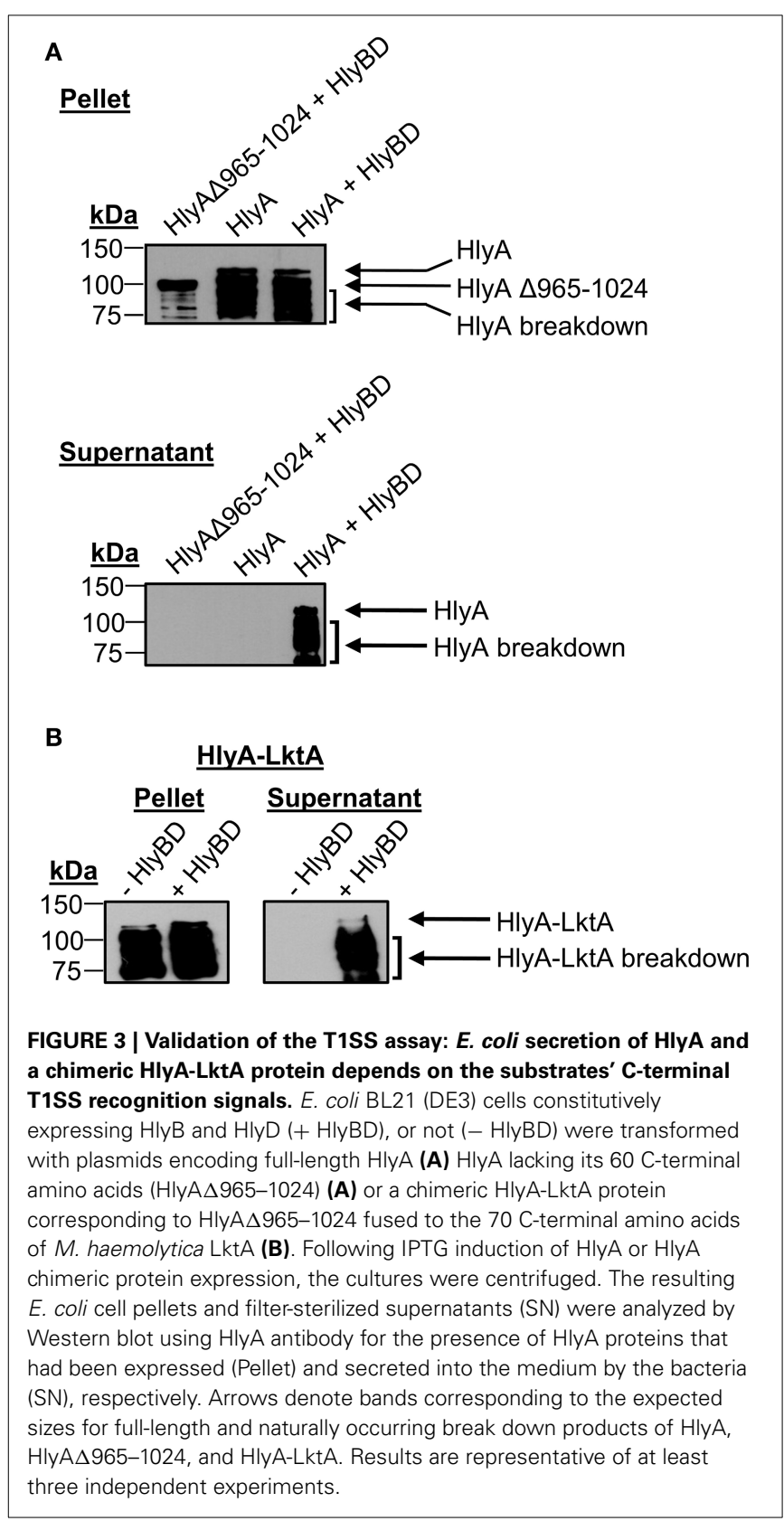

GFP-Ank13 localized predominantly in host cell nuclei but also was present in the cytosol. GFP-Ank17, GFP-Ank18, and GFP alone exhibited diffuse cytosolic patterns. To ensure that the GFP tag itself did not alter Ank subcellular trafficking, all Anks except for Ank10_01 and Ank14 were also expressed as N-terminal Flag-tag fusions and their fluorescence patterns in HeLa cells were assessed. Furthermore, to verify that fusion tag placement did not alter Ank subcellular trafficking, Ank4_01, Ank9, and Ank17 were expressed as C-terminal Flag-tagged forms. FlagBAP served as an irrelevant bacterial protein control. $\mathrm{N}$ - and C-terminal Flag-tagged fusion Anks exhibited fluorescence patterns that were similar to those of their GFP-tagged counterparts (Figure 6B and data not shown). Thus, ectopically expressed O. tsutsugamushi Anks display diverse subcellular localization patterns, the most common of which was perinuclear/ reticulate.

\section{MULTIPLE 0. TSUTSUGAMUSHI ANKS LOCALIZE TO THE ENDOPLASMIC RETICULUM (ER)}

Given that multiple GFP-Anks yielded perinuclear or reticulatelike staining patterns reminiscent of the ER, we examined if the ectopically expressed Anks colocalized with ER markers. HeLa cells expressing GFP- or Flag-tagged Anks were screened with antibody against the ER lumenal protein, calreticulin, or the ER transmembrane protein, calnexin. GFP-tagged Ank1_ 02, Ank4_01, Ank5_01, Ank9, Ank16, and Ank20 exhibited varied degrees of colocalization with both calnexin and calreticulin (Figures 7A,B; Table A8). GFP-tagged Ank2, Ank3_08, Ank10_01, and Ank11 colocalized exclusively with calreticulin, while GFP-Ank18 and GFP-Ank19 colocalized only with calnexin. Neither GFP-tagged nor Flag-tagged versions of Ank8 and Ank15 colocalized with calreticulin or calnexin. However, FlagAnk8 and Flag-Ank15 colocalized with the ER lumenal marker, protein disulfide isomerase (Figure 7C). GFP-tagged ER-tropic effectors Ank1_02, Ank2, Ank3_08, Ank4_01, Ank16, and Ank19 pronouncedly affected ER morphology, as the organelle in HeLa cells overexpressing these Anks was distended, collapsed, and/or fragmented into aggregated vesicles that were positive for calnexin/calreticulin and GFP. GFP-tagged ER-tropic effectors Ank9, Ank10_01, and Ank20 affected ER morphology to a lesser extent, as the ER staining patterns in these cells was largely of the characteristic reticulate morphology consistent with an intact ER, with some GFP- and ER marker-dual positive vesicular fragments present. GFP-tagged Ank6_02, Ank7_02, Ank12_01, Ank13, Ank14, and GFP alone neither colocalized with ER markers nor altered ER morphology (Figures 7A,B). Taken together, these data indicate that multiple ectopically expressed O. tsutsugamushi Anks localize to the ER and/or alter its morphology.

\section{O. TSUTSUGAMUSHI EXPRESSES ANK4 DURING INFECTION OF MAMMALIAN HOST CELLS}

Because all experiments performed thus far involved heterologous or ectopically expressed Anks, we evaluated if O. tsutsugamushi expressed a representative Ank during infection of mammalian host cells. We generated antiserum specific for Ank4_01 and Ank4_02 (identical paralogs; Table 2) residues 11-24. To verify the specificity of the Ank4 antiserum, Flag-tagged proteins were immunoprecipitated from whole cell lysates of HeLa cells expressing Flag-Ank4, -Ank9, or -BAP and Western blotted with Ank4 antiserum or Flag antibody. Flag antibody detected bands of the expected size for each Flag-tagged protein, thereby demonstrating that each had been expressed and precipitated (Figure 8A). Ank4 antiserum was specific for its target, as it detected Flag-Ank4 but neither Flag-Ank9 nor Flag-BAP. Confocal microscopic analysis of infected L929 cells using Ank4 antiserum in conjunction with anti-O. tsutsugamushi serum detected Ank4 signal specifically colocalized with O. tsutsugamushi organisms (Figure 8B). If secreted Ank4 was present in the infected host cells, it was at a concentration below the level of detection. These data confirm that O. tsutsugamushi translationally expresses Ank4 during infection of mammalian host cells. 


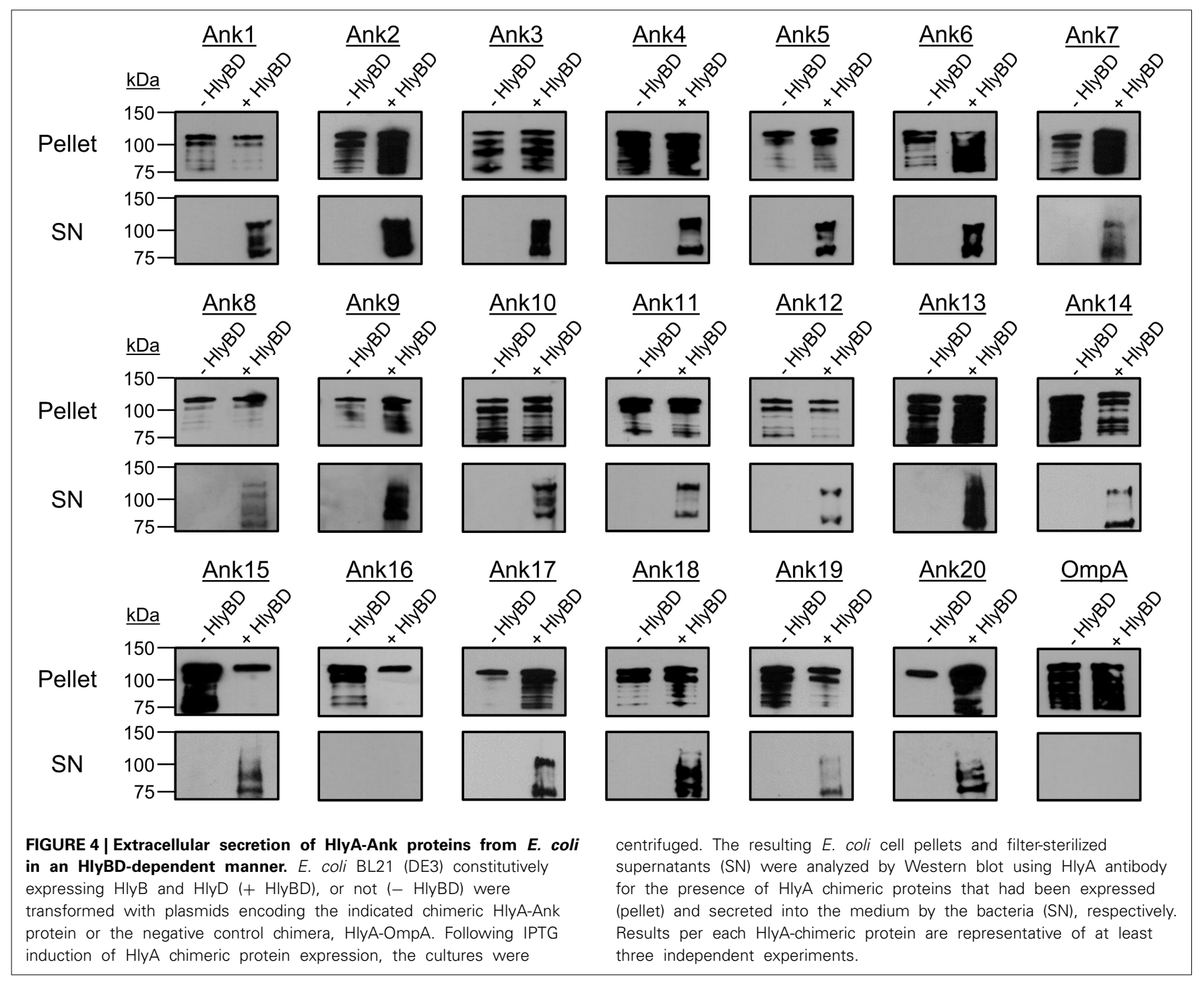

\section{DISCUSSION}

As an intracellular pathogen, O. tsutsugamushi has evolved to modulate eukaryotic host cell functions to facilitate its intracellular survival. Yet, the virulence factors that enable it to accomplish this feat are poorly defined or understood. In this study, we inferred the importance of the T1SS and family of Ank effectors to O. tsutsugamushi pathogenesis. The O. tsutsugamushi Ikeda strain expressed all T1SS component genes and all 20 distinguishable ank genes during infection of L929 cells, which implies the relevance of the encoded products. A recent transcriptional analysis of 9 of the 50 ank genes encoded by the O. tsutsugamushi Boryong strain revealed that all nine are transcribed during infection of mammalian host cells (Min et al., 2014). Thus, both O. tsutsugamushi strains express multiple ank genes during infection. Also, pooled scrub typhus patient sera was shown to weakly recognize recombinant forms of three of the nine Boryong Anks, indicating that some Anks are potentially expressed during human infection and may elicit a weak humoral immune response (Min et al., 2014). O. tsutsugamushi
Ikeda expressed Ank4 during infection of L929 cells. Because Ank4 was not detected elsewhere in infected host cells, we cannot conclude whether the bacterium translocates Ank4. However, our inability to detect secreted Ank4 is not surprising, as bacterial effectors do not need to be delivered into host cells at high abundances to perform their functions. Moreover, several lines of evidence argue for the likelihood that O. tsutsugamushi translocates Anks into host cells: (1) the O. tsutsugamushi Anks strongly resemble T1SS effectors; (2) the O. tsutsugamushi genome has retained genes encoding one of the largest Ank repertoires of any organism and the T1SS over the course of its reductive evolution (Nakayama et al., 2008; Jernigan and Bordenstein, 2014); and (3) Anks that have been examined for translocation by genetically tractable organisms using knock out-complementation approaches have proven to be bona fide effectors (Al-Khodor et al., 2008; Pan et al., 2008; Habyarimana et al., 2010; Luhrmann et al., 2010).

The T1SS translocates substrates directly into the extracellular milieu or, in the case of an intracellular bacterium such as 


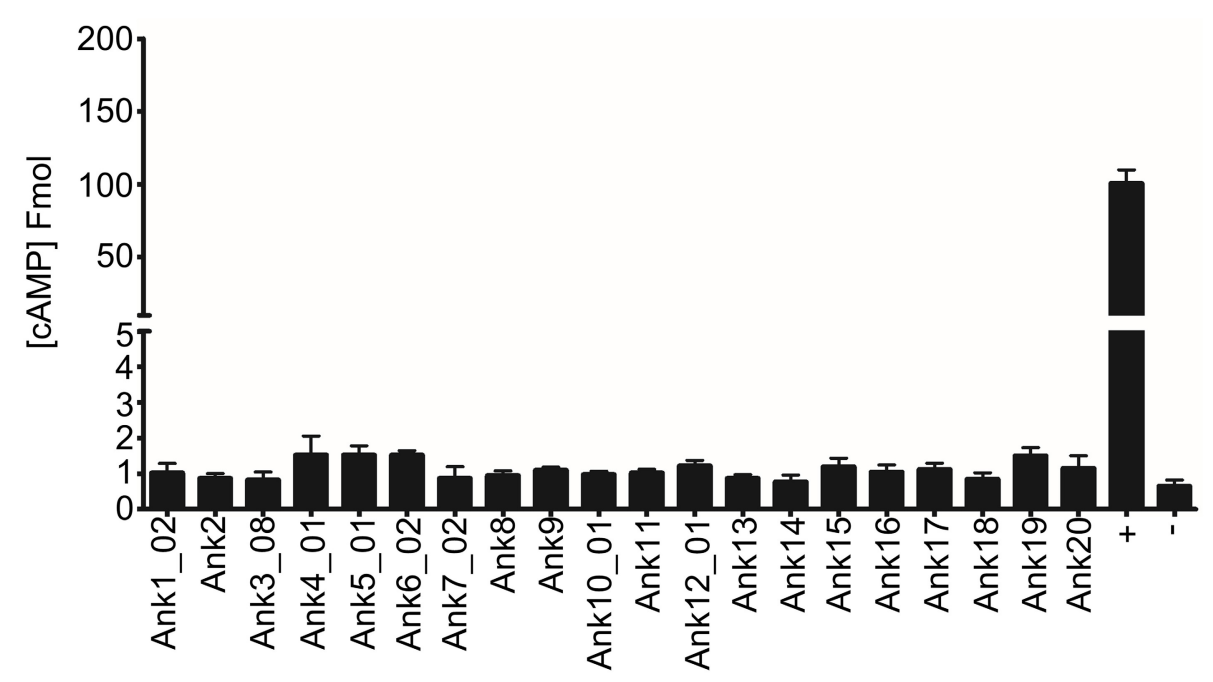

FIGURE 5 | The $C$. burnetii Dot/lcm T4SS cannot secrete

O. tsutsugamushi Anks. THP-1 macrophage-like cells were infected with C. burnetii transformants expressing CyaA-tagged O. tsutsugamushi Anks, CvpA (+), or CyaA alone (-). Bars represent the fold change in intracellular
cAMP concentration for host cells infected with wild type $C$. burnetii transformants expressing CyaA-fusions relative to control cells infected with transformants expressing CyaA alone. Results are representative of two independent experiments.
O. tsutsugamushi, directly into the host cell cytosol where the secreted effectors would have direct access to their host cell targets. T1SS secretion signals are not highly conserved, but known T1SS effectors share trends in their primary sequences (Thomas et al., 2014). In silico analyses of the Ikeda Anks identified them as potential T1SS effectors, as $37-63 \%$ of the residues in their final 60 amino acids consisted of LDAVTSIF residues, most had overall acidic pIs or had acidic C-termini, and most had very few cysteines. These sequence characteristics are similar to those of E. chaffeensis tandem repeat protein (TRP) 32, TRP47, TRP120, and Ank200, and R. typhi RARP-1 (Rickettsia ankyrin repeat protein 1), the latter two of which are Anks. These rickettsial proteins were proven to be T1SS substrates using E. coli C600 or K-12 as a heterologous host to secrete them in a TolC-dependent manner (Wakeel et al., 2011; Kaur et al., 2012). However, O. tsutsugamushi Anks were toxic to E. coli $\mathrm{C} 600$ and thus were incompatible. To circumvent this issue, we evaluated the ability of E. coli BL21 (DE3) to secrete chimeric HlyA-Ank proteins in an HlyBDdependent manner, which (1) eliminated the toxicity associated with full-length Anks; (2) avoided leaky secretion because T1SS substrates cannot be translocated from the cytosol in the absence of HlyB and HlyD (Thomas et al., 2014); and (3) enabled us to directly assess the compatibility of each Ank protein's C-terminal 60 amino acids to recognition by heterologous T1SS machinery. This approach identified 19 of the 20 distinguishable Anks as possible T1SS substrates. Only HlyA-Ank16 was not secreted. Ank16 displays characteristics that are comparable with the other T1SSpositive Anks: 52\% of its C-terminal 60 residues are LDAVTSIF amino acids, it has a pI of 5.7 , and only $1.9 \%$ of its residues are cysteines. Yet, these primary sequence characteristics alone are not enough for Ank16 to be recognized and translocated by a functionally reconstituted T1SS. Consistent with this observation, even though the final 60 amino acids of OmpA share primary sequence characteristics with O. tsutsugamushi Anks and other T1SS effectors, E. coli cannot secrete HlyA-OmpA in an HlyBD-dependent manner.

Heterologously expressed Anks could not be secreted by C. burnetii in a Dot/Icm-dependent manner. While it cannot be absolutely ruled out that this result is simply due to incompatibility of the Dot/Icm apparatus with VirB/VirD4 translocation signals, we view this possibility as being highly unlikely for three reasons. First, O. tsutsugamushi Ank C-termini more closely resemble translocation signals of T1SS vs. T4SS substrates. Second, all but one of the Ank sequences tested were compatible with a T1SS apparatus and, to our knowledge, bacterial effectors are specific for a particular secretion system. Third, Dot/Icm systems have already been proven to secrete VirB/VirD4 substrates (De Jong et al., 2008; Huang et al., 2010).

Anks of intracellular bacterial pathogens have been shown to mediate key interactions with host cell factors that aid bacterial survival. L. pneumophila AnkX prevents microtubule-dependent vesicular transport, blocking fusion of the Legionella-containing vacuole (LCV) with late endosomes (Pan et al., 2008). AnkX also posttranslationally modifies Rab1 and Rab35, which is critical for proper LCV biogenesis (Mukherjee et al., 2011). L. pneumophila AnkB co-opts host polyubiquitination machinery to facilitate bacterial survival in macrophages and amoeba, and is necessary for intrapulmonary dissemination in mice (Price et al., 2009), while AnkH and AnkJ are essential for pathogen proliferation in host cells (Habyarimana et al., 2010). C. burnetii AnkG interacts with mammalian host protein $\mathrm{p} 32$ to delay pathogen-induced apoptosis (Luhrmann et al., 2010). Other C. burnetii Anks localize to microtubules, mitochondria, and the parasitophorous vacuole membrane (Voth et al., 2009). A. phagocytophilum AnkA interacts with host cell tyrosine kinases Abl-1 and Src, which, in turn, enables its interaction with host SHP-1, an interaction that contributes to pathogen survival (Ijdo et al., 2007; Lin et al., 2007). AnkA also translocates to the host cell nucleus 


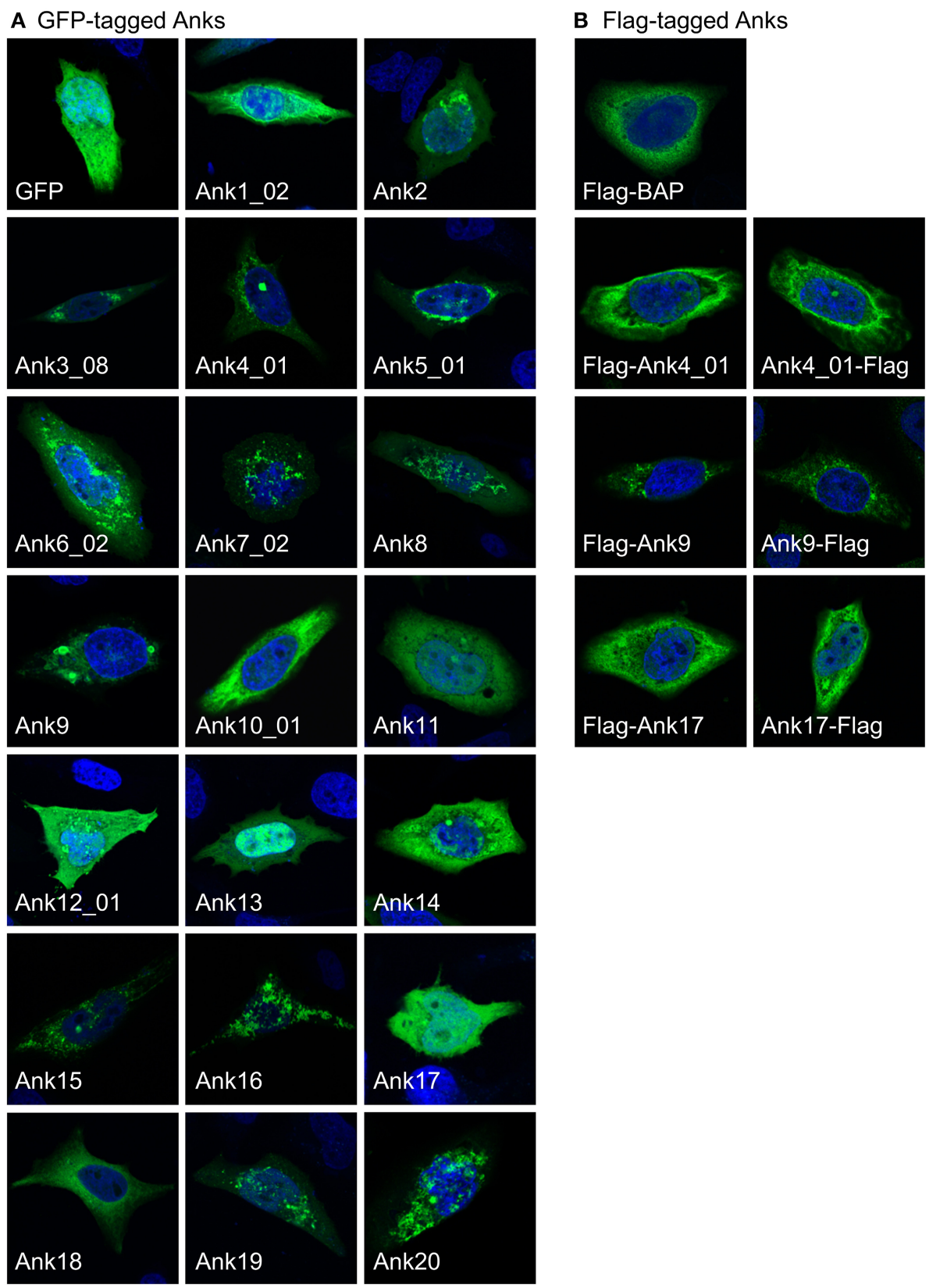

FIGURE 6 | Ectopically expressed 0 . tsutsugamushi Anks exhibit diverse subcellular localization patterns. (A) HeLa cells expressing GFP alone or the indicated Ank proteins N-terminally fused to GFP were screened with GFP antibody, and visualized using confocal microscopy. (B) Subcellular localization patterns of ectopically expressed Anks are not affected by the fusion tag itself or tag placement. HeLa cells expressing the indicated Anks as N-terminally (Flag-Ank) or C-terminally Flag-tagged (Ank-Flag) fusion proteins or Flag-BAP were screened with Flag tag antibody and examined by confocal microscopy. (A,B) HeLa cell nuclei were stained with DAPI (blue). Representative images from 2 to 4 experiments performed per each ectopically expressed Ank are presented. where it binds the promoter of the antimicrobial gene, $C Y Y B$ to inhibit host cell oxidative killing (Garcia-Garcia et al., 2009). Lastly, E. chaffeensis Ank200 also traverses to the host cell nucleus where it may globally alter host cell gene expression (Zhu et al., 2009).

To assess where O. tsutsugamushi Anks potentially traffic during infection, we examined the subcellular localization patterns of ectopically expressed Anks in HeLa cells. Of the 20 Anks evaluated, 14 trafficked to the ER and some of these promoted fragmentation, distension, and/or collapse of the organelle; one localized to the nucleus; and the other five either remained in the cytosol as diffuse or vesicular aggregates of unknown origin. Thus, O. tsutsugamushi Anks traffic to distinct subcellular locales where they likely modulate host cell processes to the pathogen's advantage. The nuclear localization of Ank13 suggests that it, like A. phagocytophilum AnkA, may globally 


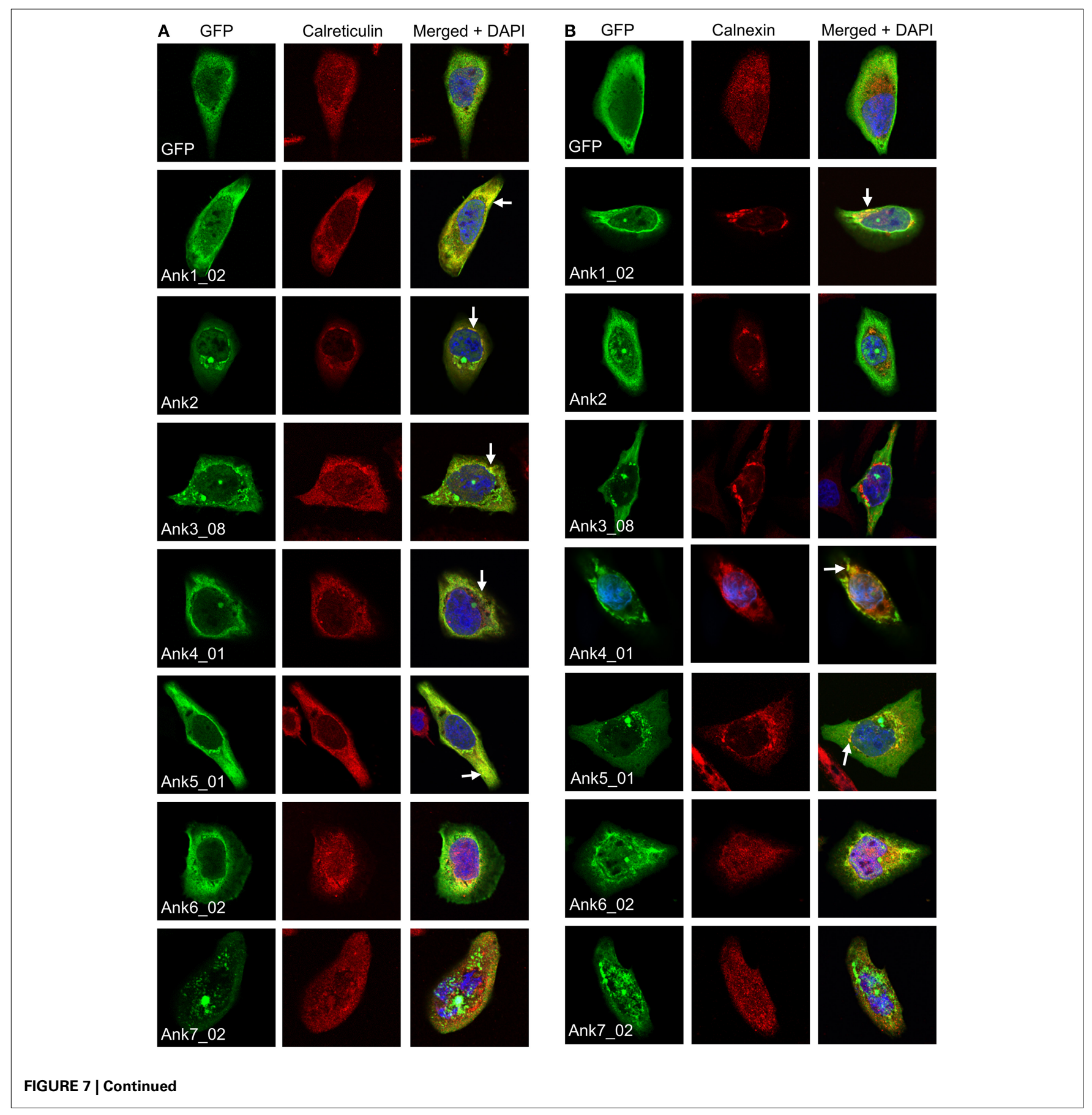

regulate host cell transcription. While many of the ER-tropic Anks accumulated in both the ER lumen and at the ER membrane, Ank3_08, Ank8, Ank10_01, Ank15, and Ank20 specifically localized in the lumen and Ank18 and Ank19 trafficked directly to the ER membrane. Thus, subsets of ER-tropic Anks may traffic to distinct locales within the ER. The mechanism responsible for these Anks' ER-tropism is unknown, as sequence analysis failed to find a KDEL ER-retention signal in any ER-tropic Ank (data not shown). Nevertheless, it can be inferred that this tropism is specific and not an artifact of overexpression because several other ectopically expressed Anks trafficked to non-ER sites.

The subcellular trafficking patterns observed for all recombinant Anks were specific to the Ank portions and not the fusion tag, as differentially tagged Anks exhibited the same localization patterns irrespective of fusion tag placement or the tag itself. Results for the Ikeda Anks obtained here largely contrast those reported for the nine ectopically expressed Boryong Anks, five of which accumulated exclusively in host cell nuclei, three of which localized to both the cytosol and 

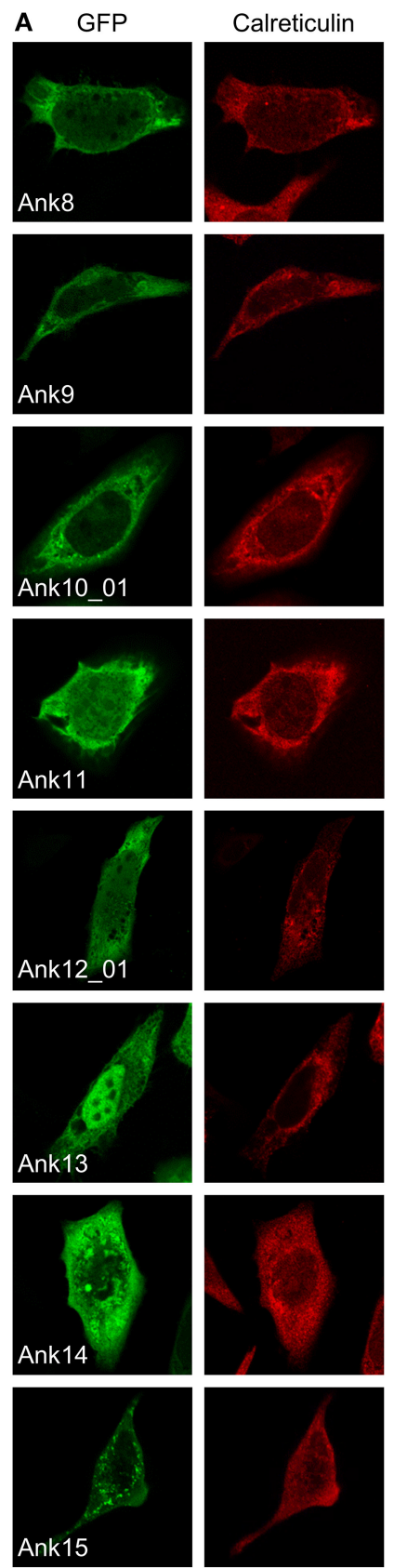
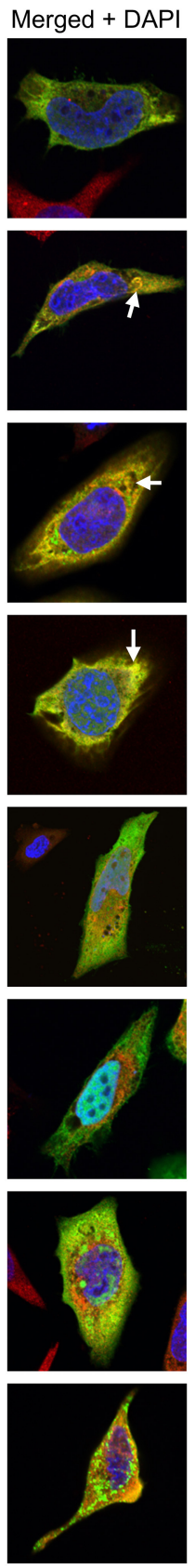
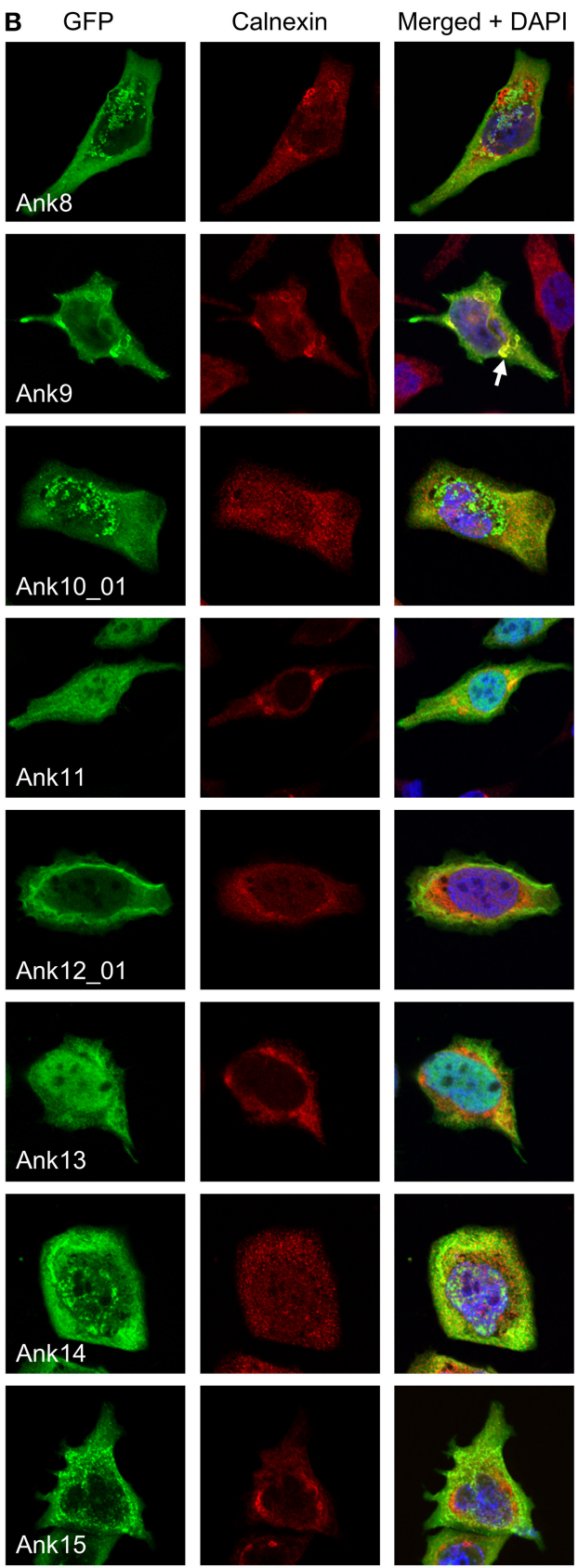

FIGURE 7 | Continued

nuclei, and one of which remained in the cytosol. It was not examined if the Boryong cytosolic Anks colocalized with ER markers, but staining for early endosomal and lysosomal markers revealed no colocalization with Anks (Min et al., 2014).

The ER is the site of protein, carbohydrate, and lipid synthesis, as well as the site of assembly of molecular complexes involved in antigen presentation (Roy, 2002). What advantage could $O$. tsutsugamushi gain by directing such a large portion of its Ank repertoire to the ER and, conversely, how might this phenomenon affect the host response to infection?
Possible clues to answer these questions arise when one considers that multiple bacterial pathogens target the ER. L. pneuomphila, Brucella spp., and Chlamydia spp. replicate in parasitophorous vacuoles that intercept ER-derived traffic, fusing with, and acquiring characteristics of ER membranes (Kagan and Roy, 2002; Celli et al., 2003; Robinson and Roy, 2006; Arasaki et al., 2012; Dumoux et al., 2012; Hubber et al., 2014). Several Brucella spp. effectors traffic to the ER, causing its structural reorganization, inducing ER stress and the unfolded protein response (UPR), and inhibition of protein secretion (Myeni et al., 2013; Smith et al., 2013). Streptococcus pyogenes injects 

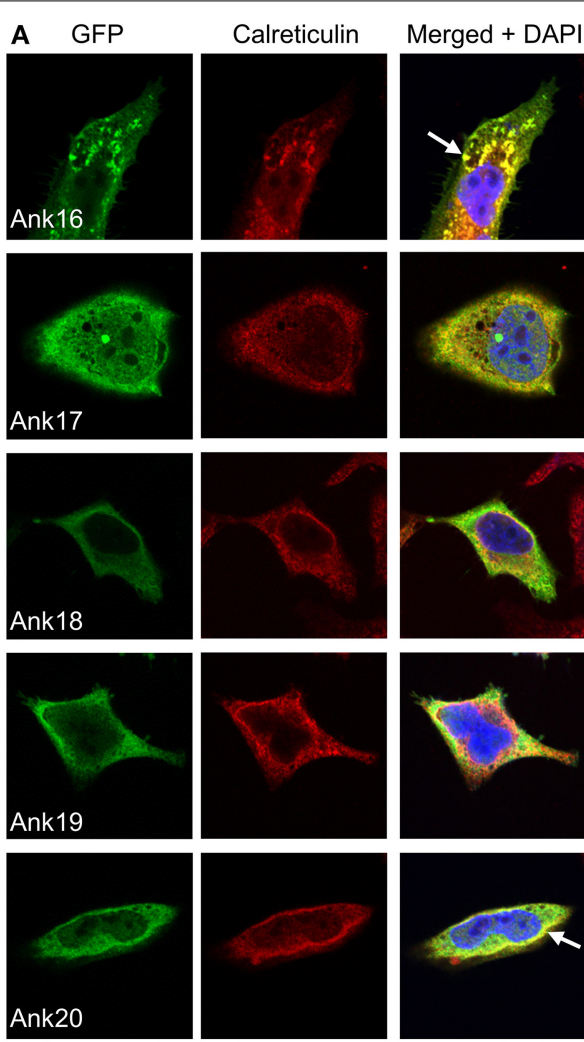

C

Flag
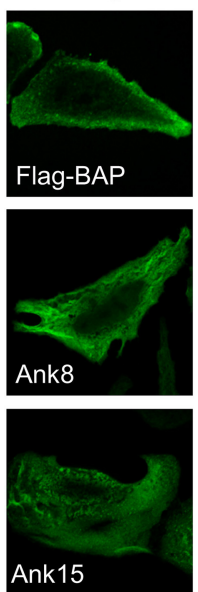

Protein disulfide isomerase
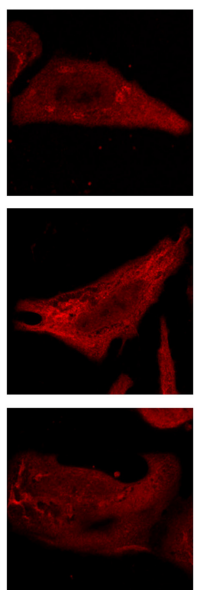
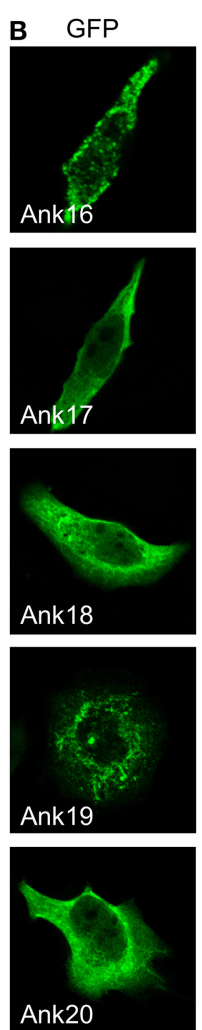

Ank20
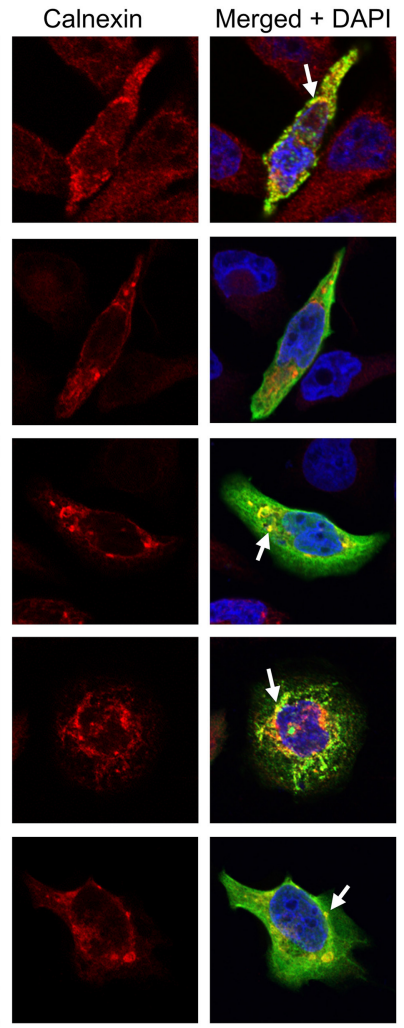

FIGURE 7 | Multiple ectopically expressed $O$. tsutsugamushi Anks localize to the endoplasmic reticulum. HeLa cells expressing GFP alone or GFP-Anks were screened with GFP antibody, and cells expressing Flag-BAP or Flag-Anks were screened with Flag antibody. Additionally, cells were stained with antibody against either of the ER lumenal markers calreticulin (A) or protein disulfide isomerase (C), or the ER transmembrane protein, calnexin (B) prior to examination by confocal microscopy. Representative fluorescence images of cells viewed for GFP (green), ER marker (red), and merged images plus DAPI (blue) are presented for each Ank from 2 to 4 independent experiments. Arrows denote representative areas of GFP and ER marker signal colocalization. toxins into host cells to generate ER stress and the UPR, which, in turn, leads to overproduction of asparagine. The released asparagine activates a $S$. pyogenes transcriptional profile that promotes bacterial growth and colonization (Baruch et al.,
2014). The Brucella melitensis effector, TcpB induces the UPR to promote bacterial survival (Smith et al., 2013). Yet, the B. abortus ER-tropic effector, VceC, which also induces the $\mathrm{UPR}$, is required for B. abortus-induced inflammatory cytokine 
A

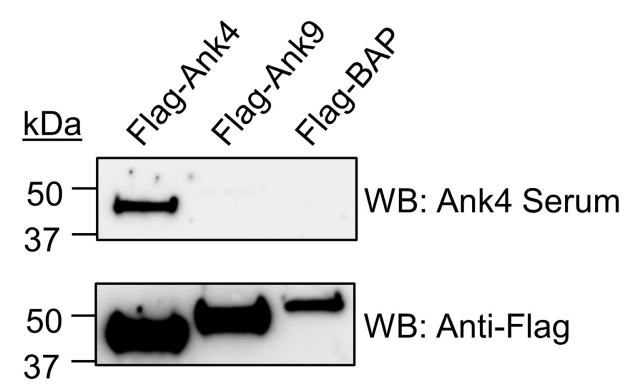

B
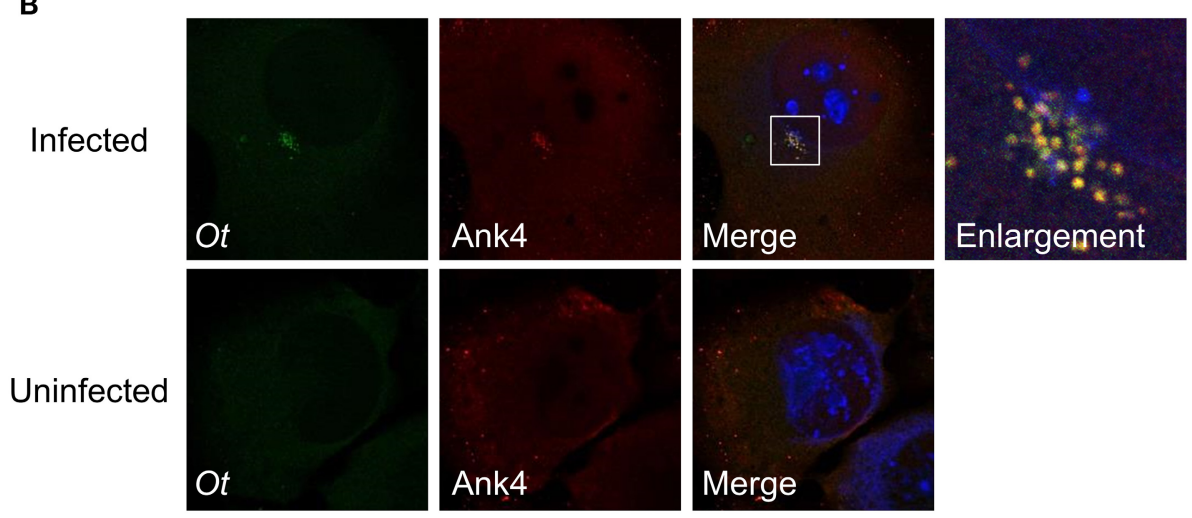

Enlargement

FIGURE 8 | O. tsutsugamushi expresses Ank4 during infection of mammalian host cells. (A) Ank4 antiserum specifically recognizes its target. Western blots of Flag-tagged Ank4, Ank9, or BAP that had been immunoprecipitated from transfected HeLa cells were screened with Ank4 antiserum and Flag tag antibody. Data presented are representative of three experiments with similar results. (B) Ank4 immunofluorescent signal colocalizes with 0 . tsutsugamushi. L929 cells infected with O. tsutsugamushi were screened with antisera specific for the bacterium (Ot; green) and Ank4 (red) were examined by laser-scanning confocal microscopy. Host cell nuclei were stained with DAPI (blue). Data presented in $(\mathbf{A})$ and $(\mathbf{B})$ are each representative of three experiments with similar results. production (Myeni et al., 2013). Likewise, the ER stress signaling pathway induced in response to Mycobacterium tuberculosis has been implicated in controlling the pathogen's growth (Lim et al., 2011). Thus, O. tsutsugamushi ER-tropic Anks conceivably mediate host-pathogen interactions that favor parasitism and/or pathogen detection and the ensuing antimicrobial response.

In closing, this study adds to the growing appreciation for the T1SS as an important translocation system for rickettsial pathogens and reveals the potential that the O. tsutsugamushi Ank armamentarium has for enabling the bacterium to parasitize mammalian host cells. Moving forward, it will be important to determine if the bacterium expresses Ank proteins in addition to Ank4 during infection and to elucidate the functional benefits that these virulence factors afford this understudied pathogen.

\section{DISCLAIMER}

The views expressed in this article are those of the authors and do not necessarily reflect the official policy or position of the Department of the Navy, Department of the Defense, or the U.S. Government. As an employee of the U.S. Government (Allen L. Richards) this work was prepared as part of his official duties and therefore because of Title 17 U.S.C paragraph 105 provides that "Copyright protection is not available for any work of the U.S. Government," this article cannot be copyrighted.

\section{ACKNOWLEDGMENTS}

We gratefully acknowledge Peter Sebo (Institute of Microbiology, Institute of Biotechnology, Academy of Sciences of the Czech Republic, Prague, Czech Republic) for providing plasmid pLG575, E. coli strain A0 34/86, and many helpful discussions. We thank Rodney Welch (University of Wisconsin, Madison, WI) for providing the HlyA antibody G3, and Marci Scidmore of Cornell University (Ithaca, NY) for providing the pEGFP-C1 vector. This work was supported by National Institutes of Health grants R03 AI101666 and R21 AI103606 (to Jason A. Carlyon), American Heart Association (AHA) Grant-in-Aid 13GRNT16810009 (to Jason A. Carlyon), AHA Predoctoral Fellowship 13PRE16840032 (to Lauren VieBrock), Military Infectious Diseases Research Program 6000.RAD1.J.A0310 (to Hong Ge and Allen L. Richards), and the Virginia Commonwealth University (VCU) Presidential Research Quest Fund (to Jason A. Carlyon). Andrea $\mathrm{R}$. Beyer is a fellowship recipient supported by VCU Institutional Research and Academic Career Development Award grant K12 GM093857. LSCM was performed at the VCU Department of Anatomy and Neurobiology Microscopy Facility, which is supported in part by funding from NIH-NINDS Center core grant 5P30NS047463. 


\section{SUPPLEMENTARY MATERIAL}

The Supplementary Material for this article can be found online at: http://www.frontiersin.org/journal/10.3389/fcimb. 2014.00186/abstract

\section{REFERENCES}

Aldick, T., Bielaszewska, M., Uhlin, B. E., Humpf, H. U., Wai, S. N., and Karch, H. (2009). Vesicular stabilization and activity augmentation of enterohaemorrhagic Escherichia coli haemolysin. Mol. Microbiol. 71, 1496-1508. doi: 10.1111/j.1365-2958.2009.06618.x

Al-Khodor, S., Price, C. T., Habyarimana, F., Kalia, A., and Abu Kwaik, Y. (2008). A Dot/Icm-translocated ankyrin protein of Legionella pneumophila is required for intracellular proliferation within human macrophages and protozoa. Mol. Microbiol. 70, 908-923. doi: 10.1111/j.1365-2958.2008.06453.x

Al-Khodor, S., Price, C. T., Kalia, A., and Abu Kwaik, Y. (2010). Functional diversity of ankyrin repeats in microbial proteins. Trends Microbiol. 18, 132-139. doi: 10.1016/j.tim.2009.11.004

Altschul, S. F., Gish, W., Miller, W., Myers, E. W., and Lipman, D. J. (1990). Basic local alignment search tool. J. Mol. Biol. 215, 403-410. doi: 10.1016/S00222836(05)80360-2

Arasaki, K., Toomre, D. K., and Roy, C. R. (2012). The Legionella pneumophila effector DrrA is sufficient to stimulate SNARE-dependent membrane fusion. Cell Host Microbe 11, 46-57. doi: 10.1016/j.chom.2011.11.009

Baruch, M., Belotserkovsky, I., Hertzog, B. B., Ravins, M., Dov, E., Mciver, K. S., et al. (2014). An extracellular bacterial pathogen modulates host metabolism to regulate its own sensing and proliferation. Cell 156, 97-108. doi: 10.1016/j.cell. 2013.12.007

Beare, P. A., Gilk, S. D., Larson, C. L., Hill, J., Stead, C. M., Omsland, A., et al. (2011). Dot/Icm type IVB secretion system requirements for Coxiella burnetii growth in human macrophages. MBio 2, e00175-e00111. doi: 10.1128/mBio.00 175-11

Bhatty, M., Laverde Gomez, J. A., and Christie, P. J. (2013). The expanding bacterial type IV secretion lexicon. Res. Microbiol. 164, 620-639. doi: 10.1016/j.resmic.2013.03.012

Campanacci, V., Mukherjee, S., Roy, C. R., and Cherfils, J. (2013). Structure of the Legionella effector AnkX reveals the mechanism of phosphocholine transfer by the FIC domain. EMBO J. 32, 1469-1477. doi: 10.1038/emboj.2013.82

Celli, J., De Chastellier, C., Franchini, D. M., Pizarro-Cerda, J., Moreno, E., and Gorvel, J. P. (2003). Brucella evades macrophage killing via VirB-dependent sustained interactions with the endoplasmic reticulum. J. Exp. Med. 198, 545-556. doi: $10.1084 /$ jem.20030088

Chang, W. H., Kang, J. S., Lee, W. K., Choi, M. S., and Lee, J. H. (1990). Serological classification by monoclonal antibodies of Rickettsia tsutsugamushi isolated in Korea. J. Clin. Microbiol. 28, 685-688.

Cho, N. H., Kim, H. R., Lee, J. H., Kim, S. Y., Kim, J., Cha, S., et al. (2007). The Orientia tsutsugamushi genome reveals massive proliferation of conjugative type IV secretion system and host-cell interaction genes. Proc. Natl. Acad. Sci. U.S.A. 104, 7981-7986. doi: 10.1073/pnas.0611553104

Christie, P. J., Whitaker, N., and Gonzalez-Rivera, C. (2014). Mechanism and structure of the bacterial type IV secretion systems. Biochim. Biophys. Acta 1843, 1578-1591. doi: 10.1016/j.bbamcr.2013.12.019

Davies, R. L., Campbell, S., and Whittam, T. S. (2002). Mosaic structure and molecular evolution of the leukotoxin operon (lktCABD) in Mannheimia (Pasteurella) haemolytica, Mannheimia glucosida, and Pasteurella trehalosi. J. Bacteriol. 184, 266-277. doi: 10.1128/JB.184.1.266-277.2002

De Jong, M. F., Sun, Y. H., Den Hartigh, A. B., Van Dijl, J. M., and Tsolis, R. M. (2008). Identification of VceA and VceC, two members of the VjbR regulon that are translocated into macrophages by the Brucella type IV secretion system. Mol. Microbiol. 70, 1378-1396. doi: 10.1111/j.1365-2958.2008.06487.x

Delepelaire, P. (2004). Type I secretion in gram-negative bacteria. Biochim. Biophys. Acta 1694, 149-161. doi: 10.1016/j.bbamcr.2004.05.001

Dumoux, M., Clare, D. K., Saibil, H. R., and Hayward, R. D. (2012). Chlamydiae assemble a pathogen synapse to hijack the host endoplasmic reticulum. Traffic 13, 1612-1627. doi: 10.1111/tra. 12002

Garcia-Garcia, J. C., Rennoll-Bankert, K. E., Pelly, S., Milstone, A. M., and Dumler, J. S. (2009). Silencing of host cell CYBB gene expression by the nuclear effector AnkA of the intracellular pathogen Anaplasma phagocytophilum. Infect. Immun. 77, 2385-2391. doi: 10.1128/IAI.00023-09
Ge, Y., and Rikihisa, Y. (2011). Subversion of host cell signaling by Orientia tsutsugamushi. Microbes Infect. 13, 638-648. doi: 10.1016/j.micinf.2011. 03.003

Habyarimana, F., Price, C. T., Santic, M., Al-Khodor, S., and Kwaik, Y. A. (2010). Molecular characterization of the Dot/Icm-translocated AnkH and AnkJ eukaryotic-like effectors of Legionella pneumophila. Infect. Immun. 78, 1123-1134. doi: 10.1128/IAI.00913-09

Huang, B., Troese, M. J., Howe, D., Ye, S., Sims, J. T., Heinzen, R. A., et al. (2010). Anaplasma phagocytophilum APH_0032 is expressed late during infection and localizes to the pathogen-occupied vacuolar membrane. Microb. Pathog. 49, 273-284. doi: 10.1016/j.micpath.2010.06.009

Huang, L., Boyd, D., Amyot, W. M., Hempstead, A. D., Luo, Z. Q., O'connor, T. J., et al. (2011). The E Block motif is associated with Legionella pneumophila translocated substrates. Cell. Microbiol. 13, 227-245. doi: 10.1111/j.14625822.2010.01531.x

Hubber, A., Arasaki, K., Nakatsu, F., Hardiman, C., Lambright, D., De Camilli, P., et al. (2014). The machinery at endoplasmic reticulum-plasma membrane contact sites contributes to spatial regulation of multiple Legionella effector proteins. PLoS Pathog. 10:e1004222. doi: 10.1371/journal.ppat.1004222

Ijdo, J. W., Carlson, A. C., and Kennedy, E. L. (2007). Anaplasma phagocytophilum AnkA is tyrosine-phosphorylated at EPIYA motifs and recruits SHP-1 during early infection. Cell. Microbiol. 9, 1284-1296. doi: 10.1111/j.14625822.2006.00871.x

Jernigan, K. K., and Bordenstein, S. R. (2014). Ankyrin domains across the Tree of Life. PeerJ 2:e264. doi: 10.7717/peerj.264

Kagan, J. C., and Roy, C. R. (2002). Legionella phagosomes intercept vesicular traffic from endoplasmic reticulum exit sites. Nat. Cell Biol. 4, 945-954. doi: $10.1038 /$ ncb883

Kaur, S. J., Rahman, M. S., Ammerman, N. C., Beier-Sexton, M., Ceraul, S. M., Gillespie, J. J., et al. (2012). TolC-dependent secretion of an ankyrin repeat-containing protein of Rickettsia typhi. J. Bacteriol. 194, 4920-4932. doi: 10.1128/JB.00793-12

Larson, C. L., Beare, P. A., Howe, D., and Heinzen, R. A. (2013). Coxiella burnetii effector protein subverts clathrin-mediated vesicular trafficking for pathogen vacuole biogenesis. Proc. Natl. Acad. Sci. U.S.A. 110, E4770-E4779. doi: 10.1073/pnas.1309195110

Lim, Y. J., Choi, J. A., Choi, H. H., Cho, S. N., Kim, H. J., Jo, E. K., et al. (2011). Endoplasmic reticulum stress pathway-mediated apoptosis in macrophages contributes to the survival of Mycobacterium tuberculosis. PLoS ONE 6:e28531. doi: 10.1371/journal.pone.0028531

Lin, M., Den Dulk-Ras, A., Hooykaas, P. J., and Rikihisa, Y. (2007). Anaplasma phagocytophilum AnkA secreted by type IV secretion system is tyrosine phosphorylated by Abl-1 to facilitate infection. Cell. Microbiol. 9, 2644-2657. doi: 10.1111/j.1462-5822.2007.00985.x

Lin, M., Zhang, C., Gibson, K., and Rikihisa, Y. (2009). Analysis of complete genome sequence of Neorickettsia risticii: causative agent of Potomac horse fever. Nucleic Acids Res. 37, 6076-6091. doi: 10.1093/nar/gkp642

Lomma, M., Dervins-Ravault, D., Rolando, M., Nora, T., Newton, H. J., Sansom, F. M., et al. (2010). The Legionella pneumophila F-box protein Lpp2082 (AnkB) modulates ubiquitination of the host protein parvin B and promotes intracellular replication. Cell. Microbiol. 12, 1272-1291. doi: 10.1111/j.14625822.2010.01467.x

Luhrmann, A., Nogueira, C. V., Carey, K. L., and Roy, C. R. (2010). Inhibition of pathogen-induced apoptosis by a Coxiella burnetii type IV effector protein. Proc. Natl. Acad. Sci. U.S.A. 107, 18997-19001. doi: 10.1073/pnas.1004380107

Luo, T., Kuriakose, J. A., Zhu, B., Wakeel, A., and Mcbride, J. W. (2011). Ehrlichia chaffeensis TRP120 interacts with a diverse array of eukaryotic proteins involved in transcription, signaling, and cytoskeleton organization. Infect. Immun. 79, 4382-4391. doi: 10.1128/IAI.05608-11

Luo, T., and Mcbride, J. W. (2012). Ehrlichia chaffeensis TRP32 interacts with host cell targets that influence intracellular survival. Infect. Immun. 80, 2297-2306. doi: 10.1128/IAI.00154-12

Mackman, N., Nicaud, J. M., Gray, L., and Holland, I. B. (1985). Genetical and functional organisation of the Escherichia coli haemolysin determinant 2001. Mol. Gen. Genet. 201, 282-288. doi: 10.1007/BF00425672

Masure, H. R., Au, D. C., Gross, M. K., Donovan, M. G., and Storm, D. R. (1990). Secretion of the Bordetella pertussis adenylate cyclase from Escherichia coli containing the hemolysin operon. Biochemistry 29, 140-145. doi: 10.1021/bi0045 $3 \mathrm{a} 017$ 
Min, C. K., Kwon, Y. J., Ha, N. Y., Cho, B. A., Kim, J. M., Kwon, E. K., et al. (2014). Multiple Orientia tsutsugamushi ankyrin repeat proteins interact with SCF1 Ubiquitin Ligase complex and eukaryotic elongation factor 1 alpha. PLoS ONE 9:e105652. doi: 10.1371/journal.pone.0105652

Mukherjee, S., Liu, X., Arasaki, K., Mcdonough, J., Galan, J. E., and Roy, C. R. (2011). Modulation of Rab GTPase function by a protein phosphocholine transferase. Nature 477, 103-106. doi: 10.1038/nature10335

Myeni, S., Child, R., Ng, T. W., Kupko, J. J. III, Wehrly, T. D., Porcella, S. F., et al. (2013). Brucella modulates secretory trafficking via multiple type IV secretion effector proteins. PLoS Pathog. 9:e1003556. doi: 10.1371/journal.ppat.1003556

Nagai, H., Cambronne, E. D., Kagan, J. C., Amor, J. C., Kahn, R. A., and Roy, C. R. (2005). A C-terminal translocation signal required for Dot/Icm-dependent delivery of the Legionella RalF protein to host cells. Proc. Natl. Acad. Sci. U.S.A. 102, 826-831. doi: 10.1073/pnas.0406239101

Nakayama, K., Yamashita, A., Kurokawa, K., Morimoto, T., Ogawa, M., Fukuhara, M., et al. (2008). The whole-genome sequencing of the obligate intracellular bacterium Orientia tsutsugamushi revealed massive gene amplification during reductive genome evolution. DNA Res. 15, 185-199. doi: 10.1093/dnares/dsn011

Ohashi, N., Koyama, Y., Urakami, H., Fukuhara, M., Tamura, A., Kawamori, F., et al. (1996). Demonstration of antigenic and genotypic variation in Orientia tsutsugamushi which were isolated in Japan, and their classification into type and subtype. Microbiol. Immunol. 40, 627-638. doi: 10.1111/j.13480421.1996.tb01120.x

Omsland, A., Beare, P. A., Hill, J., Cockrell, D. C., Howe, D., Hansen, B., et al. (2011). Isolation from animal tissue and genetic transformation of Coxiella burnetii are facilitated by an improved axenic growth medium. Appl. Environ. Microbiol. 77, 3720-3725. doi: 10.1128/AEM.02826-10

Pan, X., Luhrmann, A., Satoh, A., Laskowski-Arce, M. A., and Roy, C. R. (2008). Ankyrin repeat proteins comprise a diverse family of bacterial type IV effectors. Science 320, 1651-1654. doi: 10.1126/science. 1158160

Paris, D. H., Phetsouvanh, R., Tanganuchitcharnchai, A., Jones, M., Jenjaroen, K., Vongsouvath, M., et al. (2012). Orientia tsutsugamushi in human scrub typhus eschars shows tropism for dendritic cells and monocytes rather than endothelium. PLoS Negl. Trop. Dis. 6:e1466. doi: 10.1371/journal.pntd.0001466

Paris, D. H., Shelite, T. R., Day, N. P., and Walker, D. H. (2013). Unresolved problems related to scrub typhus: a seriously neglected life-threatening disease. Am. J. Trop. Med. Hyg. 89, 301-307. doi: 10.4269/ajtmh.13-0064

Park, J., Kim, K. J., Choi, K. S., Grab, D. J., and Dumler, J. S. (2004). Anaplasma phagocytophilum AnkA binds to granulocyte DNA and nuclear proteins. Cell. Microbiol. 6, 743-751. doi: 10.1111/j.1462-5822.2004.00400.x

Ponting, C. P., Schultz, J., Milpetz, F., and Bork, P. (1999). SMART: identification and annotation of domains from signalling and extracellular protein sequences. Nucleic Acids Res. 27, 229-232. doi: 10.1093/nar/27.1.229

Price, C. T., Al-Khodor, S., Al-Quadan, T., and Abu Kwaik, Y. (2010a). Indispensable role for the eukaryotic-like ankyrin domains of the ankyrin B effector of Legionella pneumophila within macrophages and amoebae. Infect. Immun. 78, 2079-2088. doi: 10.1128/IAI.01450-09

Price, C. T., Al-Khodor, S., Al-Quadan, T., Santic, M., Habyarimana, F., Kalia, A., et al. (2009). Molecular mimicry by an F-box effector of Legionella pneumophila hijacks a conserved polyubiquitination machinery within macrophages and protozoa. PLoS Pathog. 5:e1000704. doi: 10.1371/journal.ppat.10 00704

Price, C. T., Al-Quadan, T., Santic, M., Jones, S. C., and Abu Kwaik, Y. (2010b). Exploitation of conserved eukaryotic host cell farnesylation machinery by an F-box effector of Legionella pneumophila. J. Exp. Med. 207, 1713-1726. doi: 10.1084/jem.20100771

Price, C. T., Al-Quadan, T., Santic, M., Rosenshine, I., and Abu Kwaik, Y. (2011). Host proteasomal degradation generates amino acids essential for intracellular bacterial growth. Science 334, 1553-1557. doi: 10.1126/science.1212868

Rikihisa, Y., and Lin, M. (2010). Anaplasma phagocytophilum and Ehrlichia chaffeensis type IV secretion and Ank proteins. Curr. Opin. Microbiol. 13, 59-66. doi: 10.1016/j.mib.2009.12.008

Robinson, C. G., and Roy, C. R. (2006). Attachment and fusion of endoplasmic reticulum with vacuoles containing Legionella pneumophila. Cell. Microbiol. 8, 793-805. doi: 10.1111/j.1462-5822.2005.00666.x

Rowe, G. E., Pellett, S., and Welch, R. A. (1994). Analysis of toxinogenic functions associated with the RTX repeat region and monoclonal antibody D12 epitope of Escherichia coli hemolysin. Infect. Immun. 62, 579-588.
Roy, C. R. (2002). Exploitation of the endoplasmic reticulum by bacterial pathogens. Trends Microbiol. 10, 418-424. doi: 10.1016/S0966-842X(02) 02421-6

Schultz, J., Milpetz, F., Bork, P., and Ponting, C. P. (1998). SMART, a simple modular architecture research tool: identification of signaling domains. Proc. Natl. Acad. Sci. U.S.A. 95, 5857-5864. doi: 10.1073/pnas.95.11.5857

Smith, J. A., Khan, M., Magnani, D. D., Harms, J. S., Durward, M., Radhakrishnan, G. K., et al. (2013). Brucella induces an unfolded protein response via TcpB that supports intracellular replication in macrophages. PLoS Pathog. 9:e1003785. doi: 10.1371/journal.ppat.1003785

Thomas, S., Holland, I. B., and Schmitt, L. (2014). The Type 1 secretion pathway the hemolysin system and beyond. Biochim. Biophys. Acta 1843, 1629-1641. doi: 10.1016/j.bbamcr.2013.09.017

Thompson, S. A., and Sparling, P. F. (1993). The RTX cytotoxin-related FrpA protein of Neisseria meningitidis is secreted extracellularly by meningococci and by HlyBD+ Escherichia coli. Infect. Immun. 61, 2906-2911.

Valbuena, G., and Walker, D. H. (2012). Approaches to vaccines against Orientia tsutsugamushi. Front. Cell. Infect. Microbiol. 2:170. doi: 10.3389/fcimb.2012.00170

Vergunst, A. C., Van Lier, M. C., Den Dulk-Ras, A., Stuve, T. A., Ouwehand, A., and Hooykaas, P. J. (2005). Positive charge is an important feature of the C-terminal transport signal of the VirB/D4-translocated proteins of Agrobacterium. Proc. Natl. Acad. Sci. U.S.A. 102, 832-837. doi: 10.1073/pnas. 0406241102

Voth, D. E. (2011). ThANKs for the repeat: intracellular pathogens exploit a common eukaryotic domain. Cell. Logist. 1, 128-132. doi: 10.4161/cl.1.4.18738

Voth, D. E., Beare, P. A., Howe, D., Sharma, U. M., Samoilis, G., Cockrell, D. C., et al. (2011). The Coxiella burnetii cryptic plasmid is enriched in genes encoding type IV secretion system substrates. J. Bacteriol. 193, 1493-1503. doi: 10.1128/JB.01359-10

Voth, D. E., Howe, D., Beare, P. A., Vogel, J. P., Unsworth, N., Samuel, J. E., et al. (2009). The Coxiella burnetii ankyrin repeat domain-containing protein family is heterogeneous, with C-terminal truncations that influence Dot $/ \mathrm{Icm}$-mediated secretion. J. Bacteriol. 191, 4232-4242. doi: 10.1128/JB.01656-08

Wakeel, A., Den Dulk-Ras, A., Hooykaas, P. J., and Mcbride, J. W. (2011). Ehrlichia chaffeensis Tandem repeat proteins and Ank200 are Type 1 secretion system substrates related to the repeats-in-toxin exoprotein family. Front. Cell. Infect. Microbiol. 1:22. doi: 10.3389/fcimb.2011.00022

Zhang, F., Greig, D. I., and Ling, V. (1993). Functional replacement of the hemolysin a transport signal by a different primary sequence. Proc. Natl. Acad. Sci. U.S.A. 90, 4211-4215. doi: 10.1073/pnas.90.9.4211

Zhang, F., Yin, Y., Arrowsmith, C. H., and Ling, V. (1995). Secretion and circular dichroism analysis of the C-terminal signal peptides of HlyA and LktA. Biochemistry 34, 4193-4201. doi: 10.1021/bi00013a007

Zhu, B., Nethery, K. A., Kuriakose, J. A., Wakeel, A., Zhang, X., and Mcbride, J. W. (2009). Nuclear translocated Ehrlichia chaffeensis ankyrin protein interacts with a specific adenine-rich motif of host promoter and intronic Alu elements. Infect. Immun. 77, 4243-4255. doi: 10.1128/IAI.00376-09

Conflict of Interest Statement: The authors declare that the research was conducted in the absence of any commercial or financial relationships that could be construed as a potential conflict of interest.

Received: 14 October 2014; accepted: 11 December 2014; published online: 03 February 2015.

Citation: VieBrock L, Evans SM, Beyer AR, Larson CL, Beare PA, Ge H, Singh S, Rodino KG, Heinzen RA, Richards AL and Carlyon JA (2015) Orientia tsutsugamushi ankyrin repeat-containing protein family members are Type 1 secretion system substrates that traffic to the host cell endoplasmic reticulum. Front. Cell. Infect. Microbiol. 4:186. doi: $10.3389 /$ fcimb.2014.00186

This article was submitted to the journal Frontiers in Cellular and Infection Microbiology.

This is an open-access article distributed under the terms of the Creative Commons Attribution License (CC BY). The use, distribution or reproduction in other forums is permitted, provided the original author(s) or licensor are credited and that the original publication in this journal is cited, in accordance with accepted academic practice. No use, distribution or reproduction is permitted which does not comply with these terms. 